\title{
Fischer-Clifford Matrices and Character Table of the Maximal Subgroup $\left(2^{9}:\left(L_{3}(4)\right): 2\right.$ of $U_{6}(2): 2$
}

\author{
Abraham Love Prins ${ }^{1}{ }^{1}$ and Ramotjaki Lucky Monaledi ${ }^{2}$ \\ ${ }^{1}$ Department of Mathematics and Physics, Faculty of Applied Sciences, Cape Peninsula University of Technology, P.O. Box 1906, \\ Bellville 7535, South Africa \\ ${ }^{2}$ Department of Mathematics, Faculty of Military Science, Stellenbosch University, Private Bag X2, \\ Saldanha 7395, South Africa
}

Correspondence should be addressed to Abraham Love Prins; prinsab@cput.ac.za

Received 11 October 2018; Accepted 26 December 2018; Published 25 February 2019

Academic Editor: Adolfo Ballester-Bolinches

Copyright (C) 2019 Abraham Love Prins and Ramotjaki Lucky Monaledi. This is an open access article distributed under the Creative Commons Attribution License, which permits unrestricted use, distribution, and reproduction in any medium, provided the original work is properly cited.

The automorphism group $U_{6}(2): 2$ of the unitary group $U_{6}(2) \cong F i_{21}$ has a maximal subgroup $\bar{G}$ of the form $\left(2^{9}:\left(L_{3}(4)\right): 2\right.$ of order 20643840. In this paper, Fischer-Clifford theory is applied to the split extension group $\bar{G}$ to construct its character table. Also, class fusion from $\bar{G}$ into the parent group $U_{6}(2): 2$ is determined.

\section{Introduction}

The unitary simple group $U=U_{6}(2)$ has outer automorphisms of orders 2, 3, and 6 and hence automorphism groups of the forms $U_{1}=U_{6}(2): 2, U_{2}=U_{6}(2): 3$, and $U_{3}=U_{6}(2)$ : $S_{3}$ exist for $U$ (see the ATLAS [1]). The reader is referred to [2] for more information about the construction of matrix representations for the covering and automorphism groups of $U_{6}(2)$. Recently in [3], a 3-local identification is given for the group $P S U_{6} \cong U_{6}(2)$ and its automorphism groups $P S U_{6}(2)$ : 3, $P_{S U}(2): 2$, and $P S U_{6}(2): S_{3}$. Also, we found in the ATLAS that one of the 16 maximal subgroups of $U$ is a split extension group $A$ of the type $2^{9}: L_{3}(4)$ of index 891 and has order 10321920. The group $A$ has automorphism groups of the form $A_{1}=\left(2^{9}: L_{3}(4)\right): 2, A_{2}=\left(2^{9}: L_{3}(4)\right): 3$, and $A_{3}=\left(2^{9}: L_{3}(4)\right): S_{3}$ which sit maximally inside the groups $U_{1}, U_{2}$, and $U_{3}$, respectively.

The character table of $A$ is stored in the GAP Library [4], whereas the character table of $A_{1}$ and $A_{2}$ are not yet uploaded in GAP. In this paper, the unknown Fischer-Clifford matrices [5] of $A_{1}$ and its associated character table will be constructed. Readers are referred to [6] on a survey of Fischer-Clifford theory. It is interesting to note that our group
$A_{1}$ is the stabilizer $\operatorname{Stab}_{U_{1}}\left(v_{1}\right)$ of a singular vector $v_{1} \in V_{20}(2)$, where $V_{20}(2)$ is the irreducible module of dimension 20 over the field $G F(2)$ for $U_{6}(2): 2$ (see [3]). The method of coset analysis as discussed in [7-9] will be used in the computation of the conjugacy classes of elements of the group $A_{1}$. The Fischer-Clifford matrices and character table of $A_{3}$ were determined in [10].

\section{Group $2^{9}:\left(L_{3}(4): 2\right)$}

In this section, using a suitable permutation representation of $U_{6}(2): 2$, we identify our group $A_{1}=\left(2^{9}: L_{3}(4)\right): 2$ as a split extension $2^{9}$ by $L_{3}(4): 2$ with the aid of GAP [4] and MAGMA [11]. Then with the help of MAGMA we represent $L_{3}(4): 2$ as a matrix group $G$ of degree 9 over the Galois field $G F(2)$. Since $G$ acts absolutely irreducibly on its natural module $2^{9}$, a split extension $S$ of the form $2^{9}:\left(L_{3}(4): 2\right)$ exists. Then we create $S$ as a subgroup of $S L_{10}(2)$ and show with the help of MAGMA that $A_{1}$ is indeed an isomorphic copy of $S$.

We construct $U_{1}=U_{6}(2): 2$ within GAP, using its smallest permutation representation of degree 672 found in the online ATLAS of Finite Group Representations [12]. 
Next, we use the GAP commands "MS:= ConjugacyClassesMaximalSubgroups $\left(U_{1}\right)$ ", "A1:=MS[4]", and "Size(A1)" to represent $A_{1}=\left(2^{9}:\left(L_{3}(4)\right): 2\right.$ as a permutation group on 672 points and then use this permutation representation to construct $A_{1}$ within MAGMA. Using the sequence of MAGMA commands "a,b:=ChiefSeries(A1)", "N:= a[3]", "NormalSubgroups(A1)", "IsNormal(A1,a[3])", "IsElemen$\operatorname{taryAbelian}(\mathrm{N})$ ", "C:= Complements $(\mathrm{A} 1, \mathrm{~N})$ ", "Order $(\mathrm{C}[1])$ ", "C[1] meet N", and "IsIsomorphic(C[1], $\left.L_{3}(4): 2\right)$ ", we verified that $A_{1}=\left(2^{9}: L_{3}(4)\right): 2 \cong 2^{9}:\left(L_{3}(4): 2\right)$.

Having $A_{1}$ as a permutation group on 672 points, we use the MAGMA commands "M:=GModule(A1,N)" and "M:Maximal" to represent $L_{3}(4): 2$ as a matrix group $G$ of degree 9 over the Galois field $G F(2)$. Thus we obtain the matrix group $G=\left\langle g_{1}, g_{2}\right\rangle$ having 14 conjugacy classes of elements, where $o\left(g_{1}\right)=4$ and $o\left(g_{2}\right)=14$. The generators $g_{1}$ and $g_{2}$ of $G$ are as follows:

$$
g_{1}=\left(\begin{array}{lllllllll}
1 & 0 & 0 & 0 & 0 & 1 & 1 & 1 & 0 \\
0 & 1 & 0 & 1 & 0 & 1 & 0 & 0 & 0 \\
1 & 1 & 0 & 1 & 0 & 0 & 0 & 0 & 0 \\
1 & 0 & 0 & 1 & 1 & 1 & 0 & 0 & 1 \\
0 & 0 & 1 & 1 & 1 & 1 & 1 & 1 & 1 \\
0 & 1 & 1 & 1 & 1 & 0 & 0 & 0 & 1 \\
0 & 1 & 0 & 0 & 1 & 0 & 0 & 0 & 0 \\
1 & 0 & 1 & 0 & 1 & 1 & 1 & 0 & 0 \\
0 & 1 & 0 & 0 & 1 & 0 & 1 & 0 & 1
\end{array}\right)
$$$$
g_{2}=\left(\begin{array}{lllllllll}
1 & 0 & 0 & 1 & 1 & 0 & 0 & 1 & 1 \\
1 & 1 & 0 & 0 & 0 & 1 & 1 & 1 & 1 \\
0 & 0 & 0 & 0 & 0 & 1 & 1 & 1 & 0 \\
0 & 1 & 0 & 1 & 0 & 1 & 1 & 1 & 0 \\
1 & 0 & 1 & 0 & 1 & 1 & 0 & 0 & 0 \\
1 & 1 & 1 & 1 & 1 & 0 & 1 & 1 & 1 \\
0 & 1 & 1 & 1 & 0 & 1 & 1 & 1 & 1 \\
1 & 0 & 1 & 1 & 0 & 0 & 1 & 0 & 1 \\
0 & 1 & 0 & 1 & 0 & 1 & 1 & 0 & 0
\end{array}\right) .
$$

The MAGMA command "IsAbsolutelyIrreducible(G)" tells us that the action of the matrix group $G$ on its natural module $2^{9}$ is absolutely irreducible. Thus a split extension of the type $2^{9}:\left(L_{3}(4): 2\right)$ does exist. Hence we can construct $2^{9}:\left(L_{3}(4): 2\right)$ as a subgroup $S$ of $S L_{10}(2)$ such that $S=\left\langle s_{1}, s_{2}, s_{3}\right\rangle$ and $L_{3}(4): 2=\left\langle s_{1}, s_{2}\right\rangle$, where $o\left(s_{1}\right)=4$, $o\left(s_{2}\right)=14$, and $o\left(s_{3}\right)=2$. The generators of the matrix group $S$ of degree 10 over $G F(2)$ are as follows:

$$
s_{1}=\left(\begin{array}{llllllllll}
1 & 0 & 0 & 0 & 0 & 1 & 1 & 1 & 0 & 0 \\
0 & 1 & 0 & 1 & 0 & 1 & 0 & 0 & 0 & 0 \\
1 & 1 & 0 & 1 & 0 & 0 & 0 & 0 & 0 & 0 \\
1 & 0 & 0 & 1 & 1 & 1 & 0 & 0 & 1 & 0 \\
0 & 0 & 1 & 1 & 1 & 1 & 1 & 1 & 1 & 0 \\
0 & 1 & 1 & 1 & 1 & 0 & 0 & 0 & 1 & 0 \\
0 & 1 & 0 & 0 & 1 & 0 & 0 & 0 & 0 & 0 \\
1 & 0 & 1 & 0 & 1 & 1 & 1 & 0 & 0 & 0 \\
0 & 1 & 0 & 0 & 1 & 0 & 1 & 0 & 1 & 0 \\
0 & 0 & 0 & 0 & 0 & 0 & 0 & 0 & 0 & 1 \\
0 & 0 & 0 & 1 & 1 & 0 & 0 & 1 & 1 & 0 \\
1 & 0 & 0 & 0 & 0 & 1 & 1 & 1 & 0 \\
1 & 1 & 0 & 0 & 0 & 1 & 1 & 1 & 1 & 0 \\
0 & 0 & 0 & 0 & 0 & 1 & 1 & 1 & 0 & 0 \\
0 & 1 & 0 & 1 & 0 & 1 & 1 & 1 & 0 & 0 \\
1 & 0 & 1 & 0 & 1 & 1 & 0 & 0 & 0 & 0 \\
1 & 1 & 1 & 1 & 1 & 0 & 1 & 1 & 1 & 0 \\
0 & 1 & 1 & 1 & 0 & 1 & 1 & 1 & 1 & 0 \\
0 & 0 & 0 & 0 & 0 & 1 & 0 & 0 & 0 & 0 \\
0 & 0 & 0 & 0 & 0 & 0 & 1 & 0 & 0 & 0 \\
0 & 0 & 0 & 0 & 0 & 0 & 0 & 1 & 0 & 0 \\
0 & 0 & 0 & 0 & 0 & 0 & 0 & 0 & 1 & 0 \\
1 & 0 & 0 & 0 & 0 & 0 & 0 & 0 & 0 & 1
\end{array}\right)
$$

Since we can represent $S$ and $A_{1}$ as a matrix and permutation group, respectively, we use the MAGMA command "IsIsomorphic $\left(S, A_{1}\right)$ " to confirm that $S \cong A_{1}$. Hence we can regard $A_{1}=\left(2^{9}: L_{3}(4)\right): 2$ as the split extension $S=2^{9}:\left(L_{3}(4): 2\right)$.

\section{The Conjugacy Classes of $\bar{G}=2^{9}:\left(L_{3}(4): 2\right)$}

In this section, the conjugacy classes of $\bar{G}$ are computed using the technique of coset analysis and readers are encouraged to consult $[8,9]$ for a sound theoretical background on this technique.

Throughout the remainder of this chapter, let $\bar{G}=2^{9}$ : $\left(L_{3}(4): 2\right)$ be a split extension of $N=2^{9}$ by $L_{3}(4)$ : 
TABLE 1: The values of $\chi\left(L_{3}(4): 2 \mid 2^{9}\right)$ on the different classes of $L_{3}(4): 2$.

\begin{tabular}{lcccccccccccccc}
\hline$[h]_{L_{3}(4): 2}$ & $1 A$ & $2 A$ & $2 B$ & $3 A$ & $4 A$ & $4 B$ & $4 C$ & $5 A$ & $6 A$ & $7 A$ & $7 B$ & $8 A$ & $14 A$ & $14 B$ \\
\hline$\chi\left(L_{3}(4): 3 \mid P_{1}\right)$ & 1 & 1 & 1 & 1 & 1 & 1 & 1 & 1 & 1 & 1 & 1 & 1 & 1 & 1 \\
$\chi\left(L_{3}(4): 3 \mid P_{2}\right)$ & 21 & 7 & 5 & 3 & 1 & 1 & 3 & 1 & 1 & 0 & 0 & 1 & 0 & 0 \\
$\chi\left(L_{3}(4): 3 \mid P_{3}\right)$ & 210 & 28 & 18 & 3 & 2 & 2 & 4 & 0 & 1 & 0 & 0 & 0 & 0 & 0 \\
$\chi\left(L_{3}(4): 3 \mid P_{4}\right)$ & 280 & 28 & 8 & 1 & 4 & 4 & 0 & 0 & 1 & 0 & 0 & 2 & 0 & 0 \\
\hline$k$ & 512 & 64 & 32 & 8 & 8 & 8 & 8 & 2 & 4 & 1 & 1 & 4 & 1 & 1 \\
\hline
\end{tabular}

2, where $N$ is the vector space $V_{9}(2)$ of dimension 9 over $G F(2)$ on which the linear group $G$ acts. Since $G=\left\langle g_{1}, g_{2}\right\rangle$ is represented as a matrix group, we used the MAGMA commands "O:= Orbits $(G)$ ”, “\#O”, “\#O[1]”, “\#O[2]”, \#O[3]”, and "\#O[4]" to compute the orbit lengths of the action of $G$ on $N$. We obtain 4 orbits of lengths 1,21,210, and 280 and using the MAGMA commands "P1:= Stabilizer $(G, \mathrm{O}[1,1])$ ", "P2:= Stabilizer $(G, \mathrm{O}[2,1])$ ", "P3:= Stabilizer $(G, \mathrm{O}[3,1])$ ", and "P4:= Stabilizer $(G, \mathrm{O}[4,1])$ ", we are able to compute the corresponding point stabilizers $P_{i}, i=1,2,3,4$, which are subgroups of $G$. With the aid of MAGMA and also checking the indices of the maximal subgroups of $G$ in the ATLAS, the structures of the stabilizers $P_{i}$ are identified as $P_{1}=L_{3}(4): 2$, $P_{2}=2^{4}: S_{5}, P_{3}=2^{4}:\left(2 \times S_{3}\right)$, and $P_{4}=3^{2}:\left(Q_{8} .2\right)$, where $P_{2}$ and $P_{4}$ are maximal subgroups of $P_{1}$. We should note here that the group $L_{3}(4): 2$ has two nonconjugate isomorphic maximal subgroups $L_{1}=P_{2}$ and $L_{2}$, having the same structure $2^{4}: S_{5}$. The stabilizer $P_{3}$ sits maximally in $L_{2}$. Alternatively, we can use [13] to identify the structures of the groups $P_{i}$. Since the action of $G$ on $N$ does not fix any nontrivial subspace of $2^{9}$, we have that $2^{9}$ is an irreducible module for $G$. We can readily verify this fact by using the MAGMA command "IsIrreducible $(G)$ ".

Let $\chi\left(L_{3}(4): 2 \mid 2^{9}\right)$ be the permutation character of $L_{3}(4): 2$ on the classes of $2^{9}$. Then, from methods that were developed by Mpono [14], we obtain that $\chi\left(L_{3}(4): 2 \mid 2^{9}\right)=$ $I_{P_{1}}^{P_{1}}+I_{P_{2}}^{P_{1}}+I_{P_{3}}^{P_{1}}+I_{P_{4}}^{P_{1}}=4 \times 1 a+4 \times 20 a+2 \times 35 a+45 a+45 b+$ $2 \times 64 a+2 \times 70 a$, where $I_{P_{1}}^{P_{1}}, I_{P_{2}}^{P_{1}}, I_{P_{3}}^{P_{1}}$, and $I_{P_{4}}^{P_{1}}$ are the identity characters of the point stabilizers $P_{i}, i=1,2,3,4$, induced to $G$. Note that the identity characters $I_{P_{i}}^{P_{1}}$ are identified with the permutation characters $\chi\left(L_{3}(4): 2 \mid P_{i}\right)$ of $L_{3}(4): 2$ acting on the classes of the point stabilizers $P_{i}$. We found that $I_{P_{1}}^{P_{1}}=$ $\chi\left(L_{3}(4): 3 \mid P_{1}\right)=1 a, I_{P_{2}}^{P_{1}}=\chi\left(L_{3}(4): 2 \mid P_{2}\right)=1 a+20 a, I_{P_{3}}^{P_{1}}$ $=\chi\left(L_{3}(4): 2 \mid P_{3}\right)=1 a+2 \times 20 a+35 a+64 a+70 a$, and $I_{P_{4}}^{P_{1}}$ $=\chi\left(L_{3}(4): 3 \mid P_{4}\right)=1 a+20 a+35 a+45 a+45 b+64 a+70 a$. The permutation characters $\chi\left(L_{3}(4): 2 \mid P_{i}\right)$ are written in terms of the ordinary irreducible characters of $G$. Since we have the generators $g_{1}$ and $g_{2}$ for $G$, we compute the character tables of $G$ and the $P_{i}$ 's directly in MAGMA and use these tables together with the fusion maps of the stabilizers into $G$, to compute $\chi\left(L_{3}(4): 2 \mid P_{i}\right)$ and $\chi\left(L_{3}(4): 2 \mid 2^{9}\right)$. The values of $\chi\left(L_{3}(4): 2 \mid 2^{9}\right)$ on the different classes of $G$ determine the number $k$ of fixed points of each $g \in G$ in $2^{9}$. The values of $k$ are listed in Table 1.
The values of $k$ enabled us to determine the number $f_{j}$ of orbits $Q_{i}$ 's, $1 \leq i \leq k$, which have fused together under the action of $C_{G}(g)$, for each class representative $g \in G$, to form one orbit $\triangle_{f}$. Mpono in [14] used the technique of coset analysis to develop Programmes A and B in CAYLEY [15] for the computation of the conjucacy classes of a split extension $\bar{G}=N: G$, where $N$ is an elementary abelian $p$-group for a prime $p$ on which a linear group $G$ acts. Ali [16] adapted Programmes A and B to be used in MAGMA. Programme A computes the values of the $f_{j}^{\prime} s$, whereas Programme B determines the order of the elements for each conjugacy class $[x]$ in $\bar{G}$. We obtain that $\bar{G}$ has exactly 49 conjugacy classes. The parameters $d_{j}$ and $w$ are defined in [14]. The centralizer order of each class $[x]$ of $\bar{G}$ is computed using the formula $\left|\mathrm{C}_{\bar{G}}(x)\right|=(k / f)\left|C_{G}(g)\right|$. All the information involving the conjugacy classes of $\bar{G}$ are listed in Table 2.

\section{The Inertia Groups of $\bar{G}=2^{9}:\left(L_{3}(4): 2\right)$}

Since $G$ has four orbits on $N$, then by Brauer's Theorem [17] $G$ acts on $\operatorname{Irr}(N)$ with the same number of orbits. The lengths of the 4 orbits will be $1, r, s$, and $t$ where $r+s+t=511$, with corresponding point stabilizers $H_{1}, H_{2}, H_{3}$, and $H_{4}$ as subgroups of $G$ such that $\left[G: H_{1}\right]=1,\left[G: H_{2}\right]=r,\left[G: H_{3}\right]=$ $s$, and $\left[G: H_{4}\right]=t$. We generate $G$ as a permutation group on a set of cardinality 672 within MAGMA. Then the maximal and submaximal subgroups of $G$ are computed. Now, considering the indices of these subgroups in $G$, the number of the classes of these subgroups, and also the fact that $\bar{G}$ has 49 conjugacy classes, we deduce that the action of $G$ on $\operatorname{Irr}(N)$ has orbits of lengths $1, r=21, s=210$, and $t=280$ with respective point stabilizers $H_{1}=L_{3}(4): 2, H_{2}=2^{4}: S_{5}, H_{3}=2^{4}:\left(2 \times S_{3}\right)$, and $H_{4}=3^{2}:\left(Q_{8}: 2\right)$. Thus we obtain four inertia groups $\bar{H}_{i}=2^{9}: H_{i}, i \in\{1,2,3,4\}$, for $2^{9}:\left(L_{3}(4): 2\right)$ on $\operatorname{Irr}(N)$. Alternatively, we can also determine the inertia factor groups if we let $T$ be the matrix group of dimension 9 over $G F(2)$ formed by the transpose of the generators of $G$. Then the action of $T$ on the classes of $N=2^{9}$ is the equivalent of $G$ acting on $\operatorname{Irr}(N)$. Then with the help of MAGMA or GAP, we can easily verify that the action of $T$ on $N$ has orbits of lengths $1,21,210$, and 280 with corresponding point stabilizers $T, 2^{4}: S_{5}, 2^{4}:\left(2 \times S_{3}\right)$, and $3^{2}:\left(Q_{8}: 2\right)$. The structures of $H_{2}$ and $\mathrm{H}_{4}$ have been identified by checking the indices of the maximal subgroups of $L_{3}(4): 2 \cong L_{3}(4) \cdot 2_{2}$ in the ATLAS. The structure of $\mathrm{H}_{3}$ was determined by direct computations 
TABLE 2: The conjugacy classes of elements of $\bar{G}=2^{9}:\left(L_{3}(4): 2\right)$.

\begin{tabular}{|c|c|c|c|c|c|c|c|}
\hline$[g]_{G}$ & $k$ & $f_{j}$ & $d_{j}$ & $w$ & {$[x]_{\bar{G}}$} & $\left|[x]_{\bar{G}}\right|$ & $\left|C_{\bar{G}}(x)\right|$ \\
\hline \multirow[t]{4}{*}{$1 A$} & 512 & $f_{1}=1$ & $(0,0,0,0,0,0,0,0,0)$ & $(0,0,0,0,0,0,0,0,0)$ & $1 A$ & 1 & 20643840 \\
\hline & & $f_{2}=21$ & $(0,0,0,0,1,1,0,1,1)$ & $(0,0,0,0,1,1,0,1,1)$ & $2 A$ & 21 & 983040 \\
\hline & & $f_{3}=210$ & $(1,0,0,0,0,0,0,0,0)$ & $(1,0,0,0,0,0,0,0,0)$ & $2 B$ & 210 & 98304 \\
\hline & & $f_{4}=280$ & $(1,0,1,0,1,1,1,0,1)$ & $(1,0,1,0,1,1,1,0,1)$ & $2 C$ & 280 & 73728 \\
\hline \multirow[t]{5}{*}{$2 A$} & 64 & $f_{1}=1$ & $(0,0,0,0,0,0,0,0,0)$ & $(0,0,0,0,0,0,0,0,0)$ & $2 D$ & 960 & 21504 \\
\hline & & $f_{2}=7$ & $(0,0,0,0,0,1,0,0,0)$ & $(0,0,0,0,0,0,0,0,0)$ & $2 E$ & 6720 & 3072 \\
\hline & & $f_{3}=7$ & $(0,0,0,0,0,0,0,1,1)$ & $(0,1,1,1,1,0,0,1,0)$ & $4 A$ & 6720 & 3072 \\
\hline & & $f_{4}=21$ & $(1,0,0,0,1,1,1,0,0)$ & $(0,0,0,0,1,1,1,0,0)$ & $4 B$ & 20160 & 1024 \\
\hline & & $f_{5}=28$ & $(0,0,0,0,0,0,0,0,1)$ & $(0,1,1,1,0,1,1,1,0)$ & $4 C$ & 26880 & 768 \\
\hline \multirow[t]{8}{*}{$2 B$} & 32 & $f_{1}=1$ & $(0,0,0,0,0,0,0,0,0)$ & $(0,0,0,0,0,0,0,0,0)$ & $2 F$ & 5040 & 4096 \\
\hline & & $f_{2}=1$ & $(0,0,0,0,0,0,0,1,1)$ & $(0,0,0,0,0,0,0,0,0)$ & $2 G$ & 5040 & 4096 \\
\hline & & $f_{3}=1$ & $(1,1,1,1,1,1,1,1,1)$ & $(0,1,1,1,1,0,0,0,1)$ & $4 D$ & 5040 & 4096 \\
\hline & & $f_{4}=1$ & $(1,1,0,0,0,0,0,1,1)$ & $(0,1,1,1,1,0,0,0,1)$ & $4 E$ & 5040 & 4096 \\
\hline & & $f_{5}=4$ & $(1,1,1,0,1,0,0,1,1)$ & $(0,0,1,0,0,1,1,0,0)$ & $4 F$ & 20160 & 1024 \\
\hline & & $f_{6}=8$ & $(0,1,1,1,0,1,0,1,0)$ & $(0,0,1,1,1,1,1,1,1)$ & $4 G$ & 40320 & 512 \\
\hline & & $f_{7}=8$ & $(1,1,1,1,1,0,1,1,1)$ & $(0,0,0,0,1,1,1,1,0)$ & $4 H$ & 40320 & 512 \\
\hline & & $f_{8}=8$ & $(1,0,0,0,0,0,0,0,0)$ & $(0,0,1,1,0,0,0,0,1)$ & $4 I$ & 40320 & 512 \\
\hline \multirow[t]{4}{*}{$3 A$} & 8 & $f_{1}=1$ & $(0,0,0,0,0,0,0,0,0)$ & $(0,0,0,0,0,0,0,0,0)$ & $3 A$ & 143360 & 144 \\
\hline & & $f_{2}=1$ & $(1,0,0,1,0,1,1,1,1)$ & $(1,0,0,1,0,1,1,1,1)$ & $6 A$ & 143360 & 144 \\
\hline & & $f_{3}=3$ & $(1,1,1,1,0,1,0,0,1)$ & $(1,1,1,1,0,1,0,0,1)$ & $6 B$ & 430080 & 48 \\
\hline & & $f_{4}=3$ & $(0,0,0,0,0,0,0,1,0)$ & $(1,0,0,1,1,0,1,1,0)$ & $6 C$ & 430080 & 48 \\
\hline \multirow[t]{4}{*}{$4 A$} & 8 & $f_{1}=1$ & $(0,0,0,0,0,0,0,0,0)$ & $(0,0,0,0,0,0,0,0,0)$ & $4 J$ & 80640 & 256 \\
\hline & & $f_{2}=1$ & $(0,1,0,0,1,0,0,1,1)$ & $(0,0,0,0,0,0,0,0,0)$ & $4 K$ & 80640 & 256 \\
\hline & & $f_{3}=2$ & $(0,0,1,0,0,0,0,0,0)$ & $(0,1,1,1,1,0,0,0,1)$ & $8 A$ & 161280 & 128 \\
\hline & & $f_{4}=4$ & $(1,0,1,0,1,0,1,1,1)$ & $(0,1,0,1,1,1,1,0,1)$ & $8 B$ & 322560 & 64 \\
\hline \multirow[t]{4}{*}{$4 B$} & 8 & $f_{1}=1$ & $(0,0,0,0,0,0,0,0,0)$ & $(0,0,0,0,0,0,0,0,0)$ & $4 L$ & 161280 & 128 \\
\hline & & $f_{2}=1$ & $(1,0,0,0,0,0,0,1,0)$ & $(0,0,0,0,0,0,0,0,0)$ & $4 M$ & 161280 & 128 \\
\hline & & $f_{3}=2$ & $(1,0,0,0,1,1,1,1,1)$ & $(0,0,1,1,0,0,0,1,0)$ & $8 C$ & 322560 & 64 \\
\hline & & $f_{4}=4$ & $(1,0,0,0,0,0,0,0,0)$ & $(1,1,0,1,1,0,0,1,0)$ & $8 D$ & 645120 & 32 \\
\hline \multirow[t]{6}{*}{$4 C$} & 8 & $f_{1}=1$ & $(0,0,0,0,0,0,0,0,0)$ & $(0,0,0,0,0,0,0,0,0)$ & $4 N$ & 161280 & 128 \\
\hline & & $f_{2}=1$ & $(1,0,1,1,1,0,1,1,1)$ & $(0,0,0,0,0,0,0,0,0)$ & $4 O$ & 161280 & 128 \\
\hline & & $f_{3}=1$ & $(1,0,0,0,1,1,0,1,0)$ & $(1,0,1,0,1,1,1,1,0)$ & $8 E$ & 161280 & 128 \\
\hline & & $f_{4}=1$ & $(0,1,1,1,1,1,1,1,0)$ & $(1,0,1,0,1,1,1,1,0)$ & $8 F$ & 161280 & 128 \\
\hline & & $f_{5}=2$ & $(1,0,0,0,0,0,0,0,0)$ & $(1,1,0,1,1,1,0,0,1)$ & $8 G$ & 322560 & 64 \\
\hline & & $f_{6}=2$ & $(1,0,1,0,0,1,0,0,1)$ & $(0,1,1,1,0,0,1,1,1)$ & $8 H$ & 322560 & 64 \\
\hline \multirow[t]{2}{*}{$5 A$} & 2 & $f_{1}=1$ & $(0,0,0,0,0,0,0,0,0)$ & $(0,0,0,0,0,0,0,0,0)$ & $5 A$ & 2064384 & 10 \\
\hline & & $f_{2}=1$ & $(1,0,0,0,0,0,0,0,0)$ & $(0,0,0,0,1,1,0,0,1)$ & $10 \mathrm{~A}$ & 2064384 & 10 \\
\hline \multirow[t]{4}{*}{$6 A$} & 4 & $f_{1}=1$ & $(0,0,0,0,0,0,0,0,0)$ & $(0,0,0,0,0,0,0,0,0)$ & $6 D$ & 860160 & 24 \\
\hline & & $f_{2}=1$ & $(1,0,0,0,1,0,1,1,0)$ & $(0,0,0,0,0,0,0,0,0)$ & $6 E$ & 860160 & 24 \\
\hline & & $f_{3}=1$ & $(1,0,0,1,1,0,1,1,1)$ & $(1,0,0,1,0,1,1,1,0)$ & $12 A$ & 860160 & 24 \\
\hline & & $f_{4}=1$ & $(1,1,1,0,1,0,1,1,1)$ & $(1,0,0,1,0,1,1,1,0)$ & $12 B$ & 860160 & 24 \\
\hline $7 A$ & 1 & $f_{1}=1$ & $(0,0,0,0,0,0,0,0,0)$ & $(0,0,0,0,0,0,0,0,0)$ & $7 A$ & 1474560 & 14 \\
\hline $7 B$ & 1 & $f_{1}=1$ & $(0,0,0,0,0,0,0,0,0)$ & $(0,0,0,0,0,0,0,0,0)$ & $7 B$ & 1474560 & 14 \\
\hline \multirow[t]{4}{*}{$8 A$} & 4 & $f_{1}=1$ & $(0,0,0,0,0,0,0,0,0)$ & $(0,0,0,0,0,0,0,0,0)$ & $8 I$ & 645120 & 32 \\
\hline & & $f_{2}=1$ & $(1,0,0,1,1,1,1,1,1)$ & $(0,0,0,0,0,0,0,0,0)$ & $8 J$ & 645120 & 32 \\
\hline & & $f_{3}=1$ & $(1,1,1,0,1,0,1,1,1)$ & $(0,0,0,0,0,0,0,1,1)$ & $16 A$ & 645120 & 32 \\
\hline & & $f_{4}=1$ & $(1,1,1,1,1,1,0,0,0)$ & $(0,0,0,0,0,0,0,1,1)$ & $16 B$ & 645120 & 32 \\
\hline $14 A$ & 1 & $f_{1}=1$ & $(0,0,0,0,0,0,0,0,0)$ & $(0,0,0,0,0,0,0,0,0)$ & $14 A$ & 1474560 & 14 \\
\hline $14 B$ & 1 & $f_{1}=1$ & $(0,0,0,0,0,0,0,0,0)$ & $(0,0,0,0,0,0,0,0,0)$ & $14 B$ & 1474560 & 14 \\
\hline
\end{tabular}


in MAGMA. The groups $H_{2}, H_{3}$, and $H_{4}$ are constructed from elements within $G$ and the generators are as follows:

(i) $H_{2}=\left\langle\alpha_{1}, \alpha_{2}\right\rangle, \alpha_{1} \in 3 A$, and $\alpha_{2} \in 6 A$ where

$$
\alpha_{1}=\left(\begin{array}{lllllllll}
0 & 0 & 0 & 0 & 1 & 0 & 1 & 0 & 0 \\
0 & 1 & 0 & 0 & 1 & 0 & 1 & 0 & 0 \\
1 & 0 & 0 & 1 & 0 & 0 & 1 & 1 & 1 \\
1 & 1 & 1 & 0 & 1 & 1 & 1 & 0 & 0 \\
0 & 0 & 0 & 0 & 0 & 0 & 1 & 1 & 0 \\
0 & 0 & 0 & 0 & 0 & 1 & 0 & 0 & 0 \\
1 & 0 & 0 & 0 & 1 & 0 & 0 & 1 & 0 \\
1 & 0 & 0 & 0 & 0 & 1 & 1 & 0 & 0 \\
0 & 0 & 1 & 1 & 0 & 1 & 0 & 1 & 0 \\
1 & 0 & 0 & 0 & 1 & 1 & 0 & 0 & 0 \\
0 & 1 & 1 & 1 & 1 & 0 & 0 & 1 & 0 \\
1 & 1 & 0 & 0 & 1 & 0 & 1 & 1 & 1 \\
1 & 0 & 1 & 0 & 0 & 0 & 0 & 1 & 0 \\
1 & 0 & 0 & 0 & 0 & 1 & 1 & 0 & 0 \\
0 & 0 & 0 & 0 & 0 & 1 & 0 & 0 & 0 \\
0 & 0 & 0 & 0 & 0 & 1 & 0 & 1 & 0 \\
0 & 0 & 0 & 0 & 0 & 1 & 1 & 1 & 0 \\
0 & 0 & 1 & 1 & 0 & 1 & 1 & 0 & 0
\end{array}\right),
$$

(ii) $H_{3}=\left\langle\beta_{1}, \beta_{2}\right\rangle, \beta_{1} \in 4 C$, and $\beta_{2} \in 6 A$ where

$$
\beta_{1}=\left(\begin{array}{lllllllll}
1 & 0 & 0 & 0 & 0 & 0 & 0 & 0 & 0 \\
1 & 1 & 0 & 1 & 0 & 1 & 1 & 0 & 0 \\
0 & 0 & 0 & 1 & 1 & 1 & 1 & 1 & 1 \\
0 & 0 & 1 & 1 & 1 & 1 & 0 & 1 & 1 \\
1 & 0 & 0 & 1 & 1 & 1 & 0 & 0 & 0 \\
1 & 0 & 1 & 0 & 0 & 0 & 1 & 1 & 1 \\
1 & 0 & 0 & 1 & 0 & 1 & 1 & 0 & 0 \\
1 & 1 & 0 & 1 & 0 & 1 & 1 & 0 & 1 \\
1 & 1 & 0 & 1 & 1 & 0 & 1 & 1 & 0 \\
1 & 0 & 0 & 0 & 0 & 0 & 0 & 0 & 0 \\
1 & 0 & 0 & 1 & 1 & 1 & 0 & 0 & 1 \\
1 & 0 & 0 & 1 & 1 & 1 & 1 & 1 & 1 \\
0 & 1 & 0 & 0 & 1 & 0 & 0 & 0 & 1 \\
1 & 0 & 0 & 1 & 1 & 1 & 0 & 0 & 0 \\
1 & 0 & 1 & 0 & 0 & 0 & 1 & 1 & 1 \\
0 & 0 & 1 & 1 & 0 & 1 & 0 & 1 & 1 \\
1 & 1 & 0 & 1 & 0 & 1 & 1 & 0 & 1 \\
1 & 1 & 0 & 1 & 1 & 0 & 1 & 1 & 0
\end{array}\right),
$$

(iii) $H_{4}=\left\langle\gamma_{1}, \gamma_{2}\right\rangle, \gamma_{1} \in 2 A$, adn $\gamma_{2} \in 8 A$ where

$$
\gamma_{1}=\left(\begin{array}{lllllllll}
0 & 0 & 0 & 0 & 1 & 0 & 1 & 0 & 0 \\
0 & 1 & 0 & 0 & 0 & 0 & 0 & 0 & 0 \\
1 & 0 & 0 & 1 & 1 & 0 & 0 & 1 & 1 \\
1 & 0 & 0 & 1 & 1 & 0 & 1 & 0 & 0 \\
1 & 0 & 0 & 0 & 0 & 0 & 1 & 0 & 0 \\
0 & 0 & 0 & 0 & 0 & 1 & 0 & 0 & 0 \\
0 & 0 & 0 & 0 & 0 & 0 & 1 & 0 & 0 \\
0 & 0 & 0 & 0 & 0 & 1 & 1 & 1 & 0 \\
0 & 0 & 1 & 1 & 0 & 1 & 0 & 1 & 0 \\
1 & 1 & 0 & 1 & 0 & 0 & 0 & 0 & 0 \\
1 & 1 & 0 & 0 & 0 & 1 & 1 & 1 & 1 \\
0 & 1 & 1 & 1 & 1 & 1 & 1 & 0 & 0 \\
0 & 1 & 0 & 0 & 0 & 0 & 0 & 0 & 0 \\
0 & 0 & 0 & 0 & 0 & 1 & 0 & 1 & 1 \\
0 & 0 & 0 & 0 & 1 & 1 & 0 & 0 & 1 \\
0 & 0 & 0 & 1 & 1 & 0 & 0 & 1 & 1 \\
0 & 0 & 0 & 0 & 1 & 1 & 0 & 0 & 0 \\
0 & 0 & 0 & 0 & 1 & 0 & 0 & 0 & 1
\end{array}\right),
$$

For the purpose of constructing the character table of $\bar{G}$, we use the above generators of the $H_{i}$ 's to compute their character tables.

\section{The Fusion of $H_{2}, H_{3}$, and $H_{4}$ into $G$}

We obtain the fusions of the inertia factors $\mathrm{H}_{2}, \mathrm{H}_{3}$, and $\mathrm{H}_{4}$ into $G$ by using direct matrix conjugation in $G$ and their permutation characters in $G$ of degrees 21, 210, and 280, respectively. MAGMA was used for the various computations. The fusion maps of $H_{2}, H_{3}$, and $H_{4}$ into $G$ are shown in Tables 3, 4, and 5 .

\section{The Fischer-Clifford Matrices of $2^{9}:\left(L_{3}(4): 2\right)$}

Having obtained the fusions of the inertia factors into $L_{3}(4)$ : 2 and the conjugacy classes of $L_{3}(4): 2$ displayed in the format of Table 2, we can proceed to use the theory and properties discussed in [9] or [14] to help us in the construction of the Fischer-Clifford matrices of $2^{9}:\left(L_{3}(4)\right.$ : 2 ). Note that all the relations hold since $2^{9}$ is an elementary abelian group.

For example, consider the conjugacy class $2 \mathrm{~A}$ of $L_{3}(4)$ : 2. Then we obtain that $M(2 A)$ has the following form with corresponding weights attached to the rows and columns: 
TABLE 3: The fusion of $H_{2}$ into $L_{3}(4): 2$.

\begin{tabular}{|c|c|c|c|c|c|c|c|c|c|c|c|}
\hline$[h]_{\mathrm{H}_{2}}$ & $\longrightarrow$ & {$[g]_{L_{3}(4): 2}$} & {$[h]_{\mathrm{H}_{2}}$} & $\longrightarrow$ & {$[g]_{L_{3}(4): 2}$} & {$[h]_{\mathrm{H}_{2}}$} & $\longrightarrow$ & {$[g]_{L_{3}(4): 2}$} & {$[h]_{\mathrm{H}_{2}}$} & $\longrightarrow$ & {$[g]_{L_{3}(4): 2}$} \\
\hline $1 A$ & & $1 A$ & $2 C$ & & $2 B$ & $4 B$ & & $4 B$ & $5 A$ & & $5 A$ \\
\hline $2 A$ & & $2 B$ & $3 A$ & & $3 A$ & $4 C$ & & $4 C$ & $6 A$ & & $6 A$ \\
\hline $2 B$ & & $2 A$ & $4 A$ & & $4 A$ & $4 D$ & & $4 C$ & $8 A$ & & $8 A$ \\
\hline
\end{tabular}

TABLE 4: The fusion of $H_{3}$ into $L_{3}(4): 2$.

\begin{tabular}{|c|c|c|c|c|c|c|c|c|c|c|c|}
\hline$[h]_{H_{3}}$ & $\longrightarrow$ & {$[g]_{L_{3}(4): 2}$} & {$[h]_{H_{3}}$} & $\longrightarrow$ & {$[g]_{L_{3}(4): 2}$} & {$[h]_{H_{3}}$} & $\longrightarrow$ & {$[g]_{L_{3}(4): 2}$} & {$[h]_{\mathrm{H}_{3}}$} & $\longrightarrow$ & {$[g]_{L_{3}(4): 2}$} \\
\hline $1 A$ & & $1 A$ & $2 D$ & & $2 B$ & $4 A$ & & $4 C$ & $4 E$ & & $4 B$ \\
\hline $2 A$ & & $2 B$ & $2 E$ & & $2 B$ & $4 B$ & & $4 C$ & $6 A$ & & $6 A$ \\
\hline $2 B$ & & $2 A$ & $2 F$ & & $2 A$ & $4 C$ & & $4 A$ & & & \\
\hline $2 C$ & & $2 B$ & $3 A$ & & $3 A$ & $4 D$ & & $4 C$ & & & \\
\hline
\end{tabular}

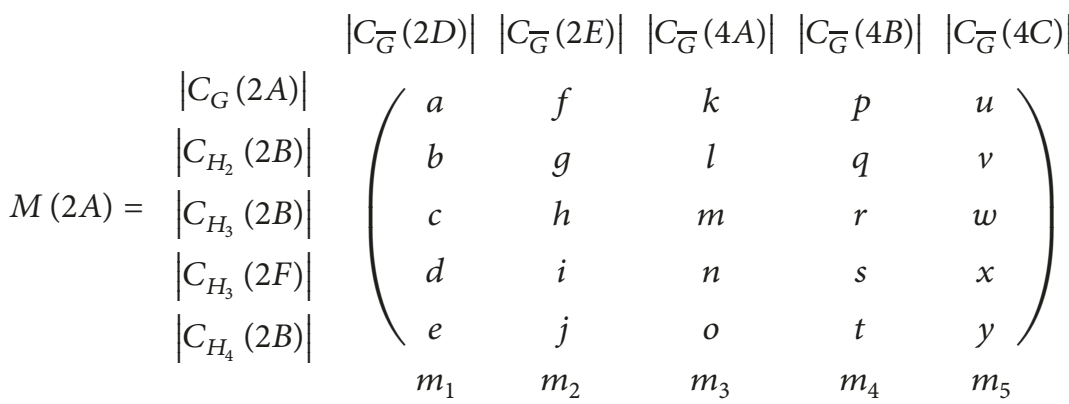

$$
\begin{aligned}
& 21504 \quad 3072 \quad 3072 \quad 1024 \quad 768 \\
& =\begin{array}{c}
336 \\
48 \\
16 \\
12
\end{array}\left(\begin{array}{ccccc}
a & f & k & p & u \\
b & g & l & q & v \\
c & h & m & r & w \\
d & i & n & s & x \\
e & j & o & t & y \\
8 & 56 & 56 & 168 & 224
\end{array}\right) .
\end{aligned}
$$

We have $a=f=k=p=u=1, b=c=7, d=21$, and $e=28$ by using Theorem 5.2.4 and property (e) of the Fischer-Clifford matrix $M(g)$ (both found in [14]). Thus we obtain the following form:

$$
\begin{aligned}
& M(2 A) \\
& \begin{array}{c}
\quad 21504 \\
336 \\
48 \\
48 \\
16 \\
12
\end{array} \quad\left(\begin{array}{ccccc}
1 & 1 & 1 & 1 & 1 \\
7 & g & l & q & v \\
7 & h & m & r & w \\
21 & i & n & s & x \\
28 & j & o & t & y \\
8 & 56 & 56 & 168 & 224
\end{array}\right) .
\end{aligned}
$$

By the orthogonality relations for columns and rows and remaining properties of the matrix $M(g)$ found in Chapter 5 of [14], we obtain the desired Fischer-Clifford matrix $M(2 A)$ of $\bar{G}$ given below:

$$
M(2 A)=\left(\begin{array}{ccccc}
1 & 1 & 1 & 1 & 1 \\
7 & -1 & -5 & 3 & -1 \\
7 & 7 & -1 & -1 & -1 \\
21 & -3 & 9 & 1 & -3 \\
28 & -4 & -4 & -4 & 4
\end{array}\right)
$$

For each class representative $g \in L_{3}(4): 2$, we construct a Fischer-Clifford matrix $M(g)$. These are listed in Table 6.

\section{Character Table of $2^{9}:\left(L_{3}(4): 2\right)$}

Having obtained the Fischer-Clifford matrices, the fusion maps of the $H_{i}$ 's into $L_{3}(4): 2$, and the character tables of the inertia factors $H_{i}$, we construct the character table 
of $2^{9}:\left(L_{3}(4): 2\right)$ following the methodology discussed in Section 5.2 of [14]. For example, we calculate the partial character table of $2^{9}:\left(L_{3}(4): 2\right)$ corresponding to the coset of $2 A \in L_{3}(4): 2$. From the Fischer-Clifford matrix $M(2 A)$ we obtain that

$$
\begin{aligned}
M_{1}(2 A) & =\left(\begin{array}{lllll}
1 & 1 & 1 & 1 & 1
\end{array}\right), \\
M_{2}(2 A) & =\left(\begin{array}{lllll}
7 & -1 & -5 & 3 & -1
\end{array}\right), \\
M_{3}(2 A) & =\left(\begin{array}{ccccc}
7 & 7 & -1 & -1 & -1 \\
21 & -3 & 9 & 1 & -3
\end{array}\right) \\
\text { and } M_{4}(2 A) & =\left(\begin{array}{lllll}
28 & -4 & -4 & -4 & 4
\end{array}\right) .
\end{aligned}
$$

Let $C_{1}(2 A), C_{2}(2 A), C_{3}(2 A)$, and $C_{4}(2 A)$ be the partial character tables of the inertia factors for the classes which fuse to $2 A \in L_{3}(4): 2$. Then the partial character table of $2^{9}$ : $\left(L_{3}(4): 2\right)$ on the classes $\{2 D, 2 E, 4 A, 4 B, 4 C\}$ is given by

$$
C_{1}(2 A) M_{1}(2 A)=\left(\begin{array}{c}
1 \\
-1 \\
-6 \\
6 \\
-7 \\
7 \\
-3 \\
-3 \\
3 \\
3 \\
8 \\
-8 \\
0 \\
0
\end{array}\right)\left(\begin{array}{lllll}
1 & 1 & 1 & 1 & 1
\end{array}\right)
$$

$$
=\left(\begin{array}{ccccc}
1 & 1 & 1 & 1 & 1 \\
-1 & -1 & -1 & -1 & -1 \\
-6 & -6 & -6 & -6 & -6 \\
6 & 6 & 6 & 6 & 6 \\
-7 & -7 & -7 & -7 & -7 \\
7 & 7 & 7 & 7 & 7 \\
-3 & -3 & -3 & -3 & -3 \\
-3 & -3 & -3 & -3 & -3 \\
3 & 3 & 3 & 3 & 3 \\
3 & 3 & 3 & 3 & 3 \\
8 & 8 & 8 & 8 & 8 \\
-8 & -8 & -8 & -8 & -8 \\
0 & 0 & 0 & 0 & 0 \\
0 & 0 & 0 & 0 & 0
\end{array}\right)
$$

$C_{2}(2 A) M_{2}(2 A)=\left(\begin{array}{c}1 \\ -1 \\ 2 \\ -2 \\ -1 \\ 1 \\ 0 \\ -3 \\ -3 \\ 3 \\ 3 \\ 0\end{array}\right)\left(\begin{array}{lllll}7 & -1 & -5 & 3 & -1\end{array}\right)$

$$
=\left(\begin{array}{ccccc}
7 & -1 & -5 & 3 & -1 \\
-7 & 1 & 5 & -3 & 1 \\
14 & -2 & -10 & 6 & -2 \\
-14 & 2 & 10 & -6 & 2 \\
-7 & 1 & 5 & -3 & 1 \\
7 & -1 & -5 & 3 & -1 \\
0 & 0 & 0 & 0 & 0 \\
-21 & 3 & 15 & -9 & 3 \\
-21 & 3 & 15 & -9 & 3 \\
21 & -3 & -15 & 9 & -3 \\
21 & -3 & -15 & 9 & -3 \\
0 & 0 & 0 & 0 & 0
\end{array}\right)
$$

$C_{3}(2 A) M_{3}(2 A)$

$$
=\left(\begin{array}{cc}
1 & 1 \\
-1 & -1 \\
1 & -1 \\
-1 & 1 \\
-2 & 0 \\
2 & 0 \\
3 & -1 \\
-3 & -1 \\
3 & 1 \\
-3 & 1 \\
0 & 0 \\
0 & 0 \\
0 & -2 \\
0 & 2
\end{array}\right)\left(\begin{array}{ccccc}
7 & 7 & -1 & -1 & -1 \\
21 & -3 & 9 & 1 & -3
\end{array}\right)
$$




$$
=\left(\begin{array}{ccccc}
28 & 4 & 8 & 0 & -4 \\
-28 & -4 & -8 & 0 & 4 \\
-14 & 10 & -10 & -2 & 2 \\
14 & -10 & 10 & 2 & -2 \\
-14 & -14 & 2 & 2 & 2 \\
14 & 14 & -2 & -2 & -2 \\
0 & 24 & -12 & -4 & 0 \\
-42 & -18 & -6 & 2 & 6 \\
42 & 18 & 6 & -2 & -6 \\
0 & -24 & 12 & 4 & 0 \\
0 & 0 & 0 & 0 & 0 \\
0 & 0 & 0 & 0 & 0 \\
-42 & 6 & -18 & -2 & 6 \\
42 & -6 & 18 & 2 & -6
\end{array}\right)
$$

Similarly, the partial character table associated with each coset $\mathrm{Ng}$ is computed. If necessary, we will restrict some characters of $\operatorname{Irr}\left(U_{6}(2): 2\right)$ to $\bar{G}$, to ensure that each partial character table corresponding to a coset $\mathrm{Ng}$ will give rise to the desired set $\operatorname{Irr}(\bar{G})$.

The character table of $\bar{G}$ will be partitioned row-wise into 4 blocks $\Delta_{1}, \Delta_{2}, \Delta_{3}$, and $\Delta_{4}$ where each block corresponds to an inertia group $\bar{H}_{i}=2^{9}: H_{i}$. Therefore $\operatorname{Irr}\left(2^{9}:\left(L_{3}(4)\right.\right.$ : 2)) $=\bigcup_{i=1}^{4} \Delta_{i}$, where $\Delta_{1}=\left\{\chi_{j} \mid 1 \leq j \leq 14\right\}, \Delta_{2}=\left\{\chi_{j} \mid\right.$

\begin{tabular}{|c|c|c|c|c|c|}
\hline$[h]_{H_{4}} \longrightarrow$ & {$[g]_{L_{3}(4): 2}$} & {$[h]_{H_{4}} \longrightarrow$} & {$[g]_{L_{3}(4): 2}$} & {$[h]_{H_{4}}$} & $\longrightarrow[g]_{L_{3}(4): 2}$ \\
\hline $1 A$ & $1 A$ & $3 A$ & $3 A$ & $6 A$ & $6 A$ \\
\hline $2 A$ & $2 B$ & $4 A$ & $4 A$ & $8 A$ & $8 A$ \\
\hline $2 B$ & $2 A$ & $4 B$ & $4 B$ & $8 B$ & $8 A$ \\
\hline
\end{tabular}
$15 \leq j \leq 26\}, \Delta_{3}=\left\{\chi_{j} \mid 27 \leq j \leq 40\right\}$, and $\Delta_{4}=\left\{\chi_{j} \mid 41 \leq\right.$
TABLE 5: The fusion of $H_{4}$ into $L_{3}(4): 2$.

$j \leq 49\}$. The character table of $2^{9}:\left(L_{3}(4): 2\right)$ is shown in Table 7. The consistency and accuracy of the character table of $2^{9}:\left(L_{3}(4): 2\right)$ have been tested by using the GAP code labelled as Programme E in [18].

The information about the conjugacy classes found in Table 2 can be used to compute the power maps for the elements of $\bar{G}$ and then with the aid of Programme E in [18] we can verify that we obtained the unique $p$-power maps listed in Table 8 for our Table 7.

\section{The Fusion of $2^{9}:\left(L_{3}(4): 2\right)$ into $U_{6}(2): 2$}

Since $\bar{G}$ is a maximal subgroup of $U_{6}(2): 2$ of index 891, then the action of $U_{6}(2): 2$ on the cosets of $\bar{G}$ gives rise to a permutation character $\chi\left(U_{6}(2): 2 \mid \bar{G}\right)$ of degree 891 . We deduce from the character table of $U_{6}(2): 2$ found in GAP that $\chi\left(U_{6}(2): 2 \mid \bar{G}\right)=1 a+22 a+252 a+616 a$, where $1 a$, $22 a, 252 a$, and $616 a$ are irreducible characters of $U_{6}(2): 2$ of degrees 1, 22, 252, and 616, respectively.

We are able to obtain the partial fusion of $\bar{G}$ into $U_{6}(2): 2$, using the information provided by the values of $\chi\left(U_{6}(2): 2\right.$ | $\bar{G})$ on the classes of $\bar{G}$ and the power maps of $\bar{G}$ and $U_{6}(2): 2$. Then, the technique of set intersections for characters (see $[9,14,19])$ is applied to restrict some ordinary irreducible characters of $U_{6}(2): 2$ of small degrees to $\bar{G}$, to determine fully the fusion of the classes of $\bar{G}$ into $U_{6}(2): 2$.

Let $\zeta$ be the character afforded by the regular representation of $L_{3}(4): 2$. We obtain that $\zeta=\sum_{i=1}^{14} \alpha_{i} \Phi_{i}$, where $\Phi_{i} \epsilon$ $\operatorname{Irr}\left(L_{3}(4): 2\right)$ and $\alpha_{i}=\operatorname{deg}\left(\Phi_{i}\right)$. Then $\zeta$ can be regarded as a character of $2^{9}:\left(L_{3}(4): 2\right)$ which contains $2^{9}$ in its kernel such that

$$
\zeta(x)= \begin{cases}\left|L_{3}(4): 2\right| & \text { if } x \in 2^{9} \\ 0 & \text { otherwise. }\end{cases}
$$

If $\phi$ is a character of $U_{6}(2): 2$ than we have that

$$
\begin{aligned}
& \langle\zeta, \phi\rangle_{\bar{G}}=\frac{1}{\left|2^{9}:\left(L_{3}(4): 2\right)\right|}\{\zeta(1 A) \phi(1 A) \\
& +21 \zeta(2 A) \phi(2 A)+210 \zeta(2 B) \phi(2 B)+280 \zeta(2 C) \\
& \cdot \phi(2 C)\}=\frac{1}{\mid 2^{9}:\left(L_{3}(4): 2 \mid\right.}\left\{\left|L_{3}(4): 2\right|\right. \\
& \cdot(\phi(1 A)+21 \phi(2 A)+210 \phi(2 B)+280 \phi(2 C))\} \\
& =\frac{1}{512}\{\phi(1 A)+21 \phi(2 A)+210 \phi(2 B) \\
& +280 \phi(2 C)\}=\left\langle\phi_{2^{9}}, 1_{2^{9}}\right\rangle .
\end{aligned}
$$


TABLE 6: The Fischer-Clifford matrices of $2^{9}:\left(L_{3}(4): 2\right)$.

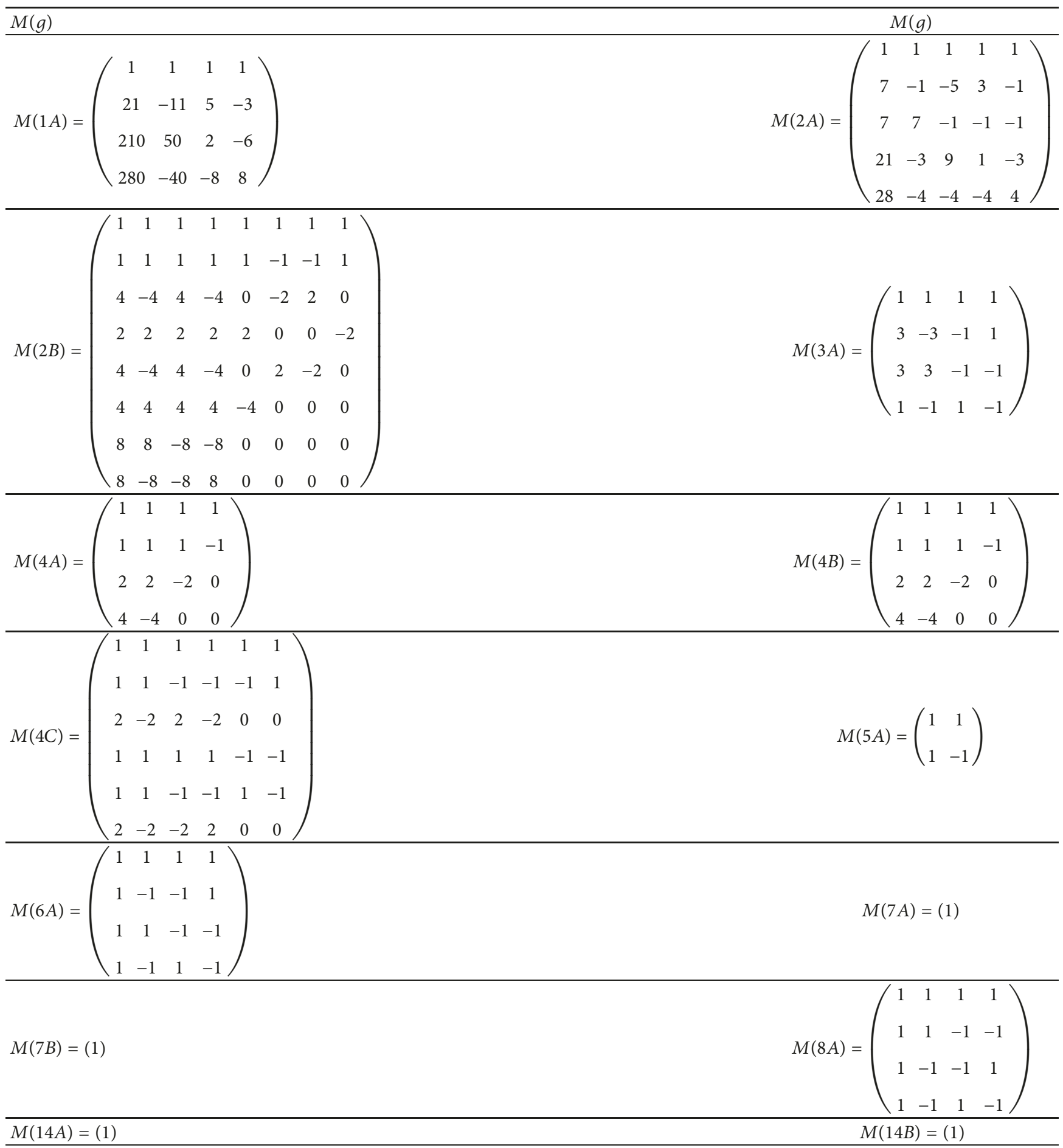

Here $1_{2^{9}}$ is the identity character of $2^{9}$ and $\phi_{2^{9}}$ is the restriction of $\phi$ to $2^{9}$. We obtain that

$$
\phi_{2^{9}}=a_{1} \theta_{1}+a_{2} \theta_{2}+a_{3} \theta_{3}+a_{4} \theta_{4},
$$

where $a_{i} \in \mathbb{N} \cup\{0\}$ and $\theta_{i}$ are the sums of the irreducible characters of $2^{9}$ which are in the same orbit under the action of $L_{3}(4): 2$ on $\operatorname{Irr}\left(2^{9}\right)$, for $i \in\{1,2,3,4\}$. Let $\varphi_{j} \in \operatorname{Irr}\left(2^{9}\right)$, where $j \in\{1,2,3, \ldots, 512\}$. Then we obtain that

$$
\begin{aligned}
& \theta_{1}=\varphi_{1}, \quad \operatorname{deg}\left(\theta_{1}\right)=1 \\
& \theta_{2}=\sum_{j=2}^{22} \varphi_{j}, \quad \operatorname{deg}\left(\theta_{2}\right)=21
\end{aligned}
$$




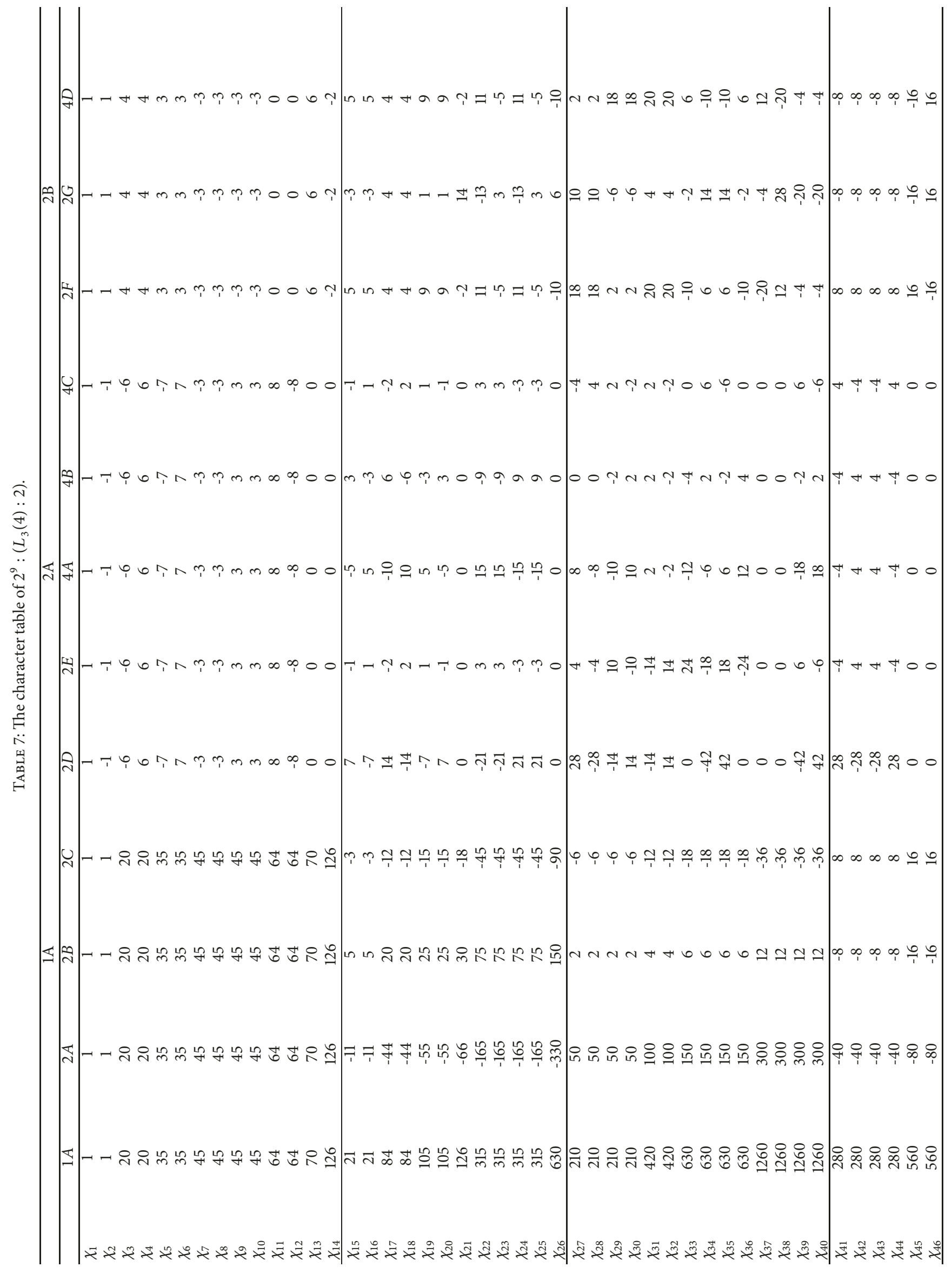




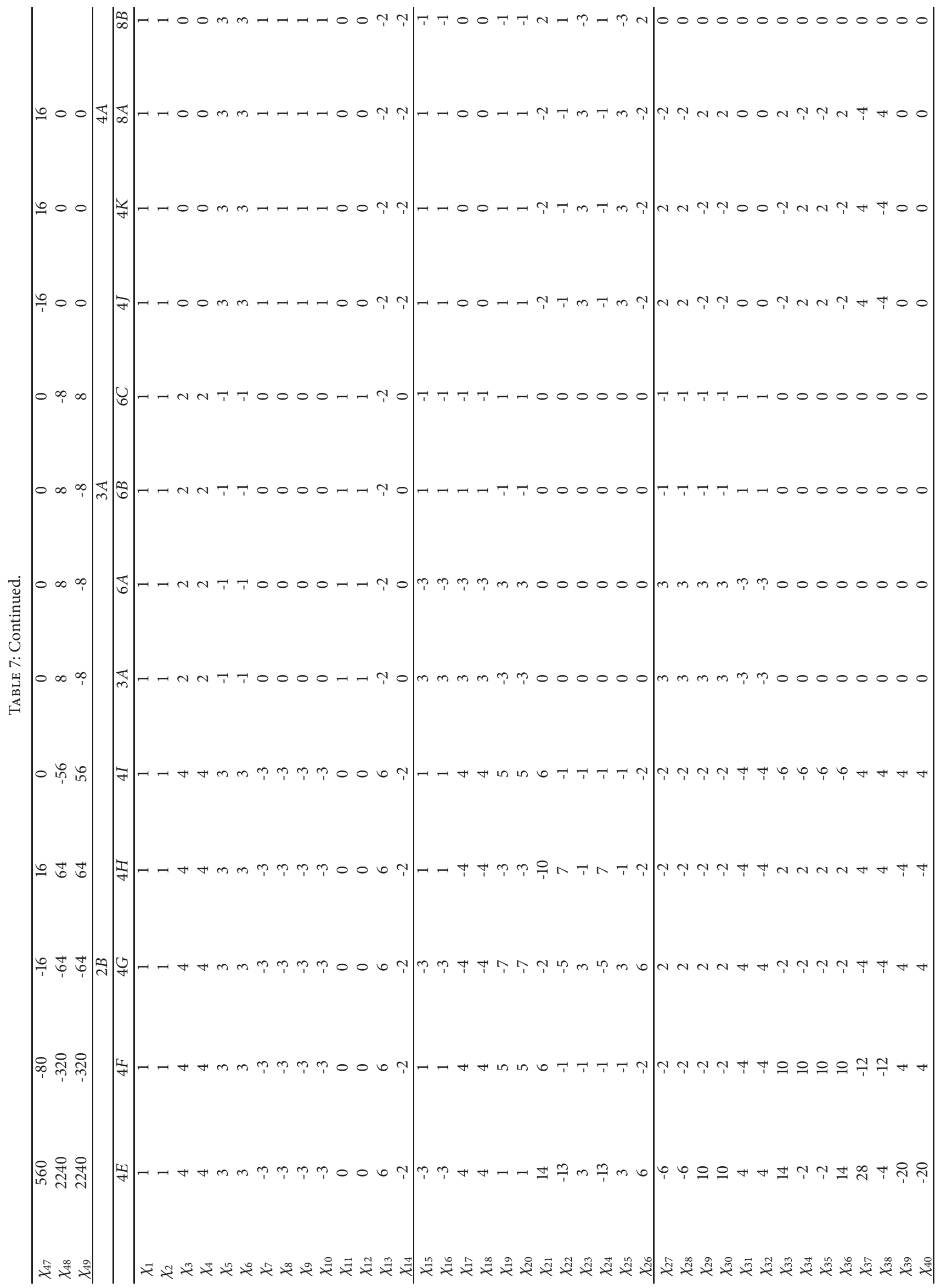




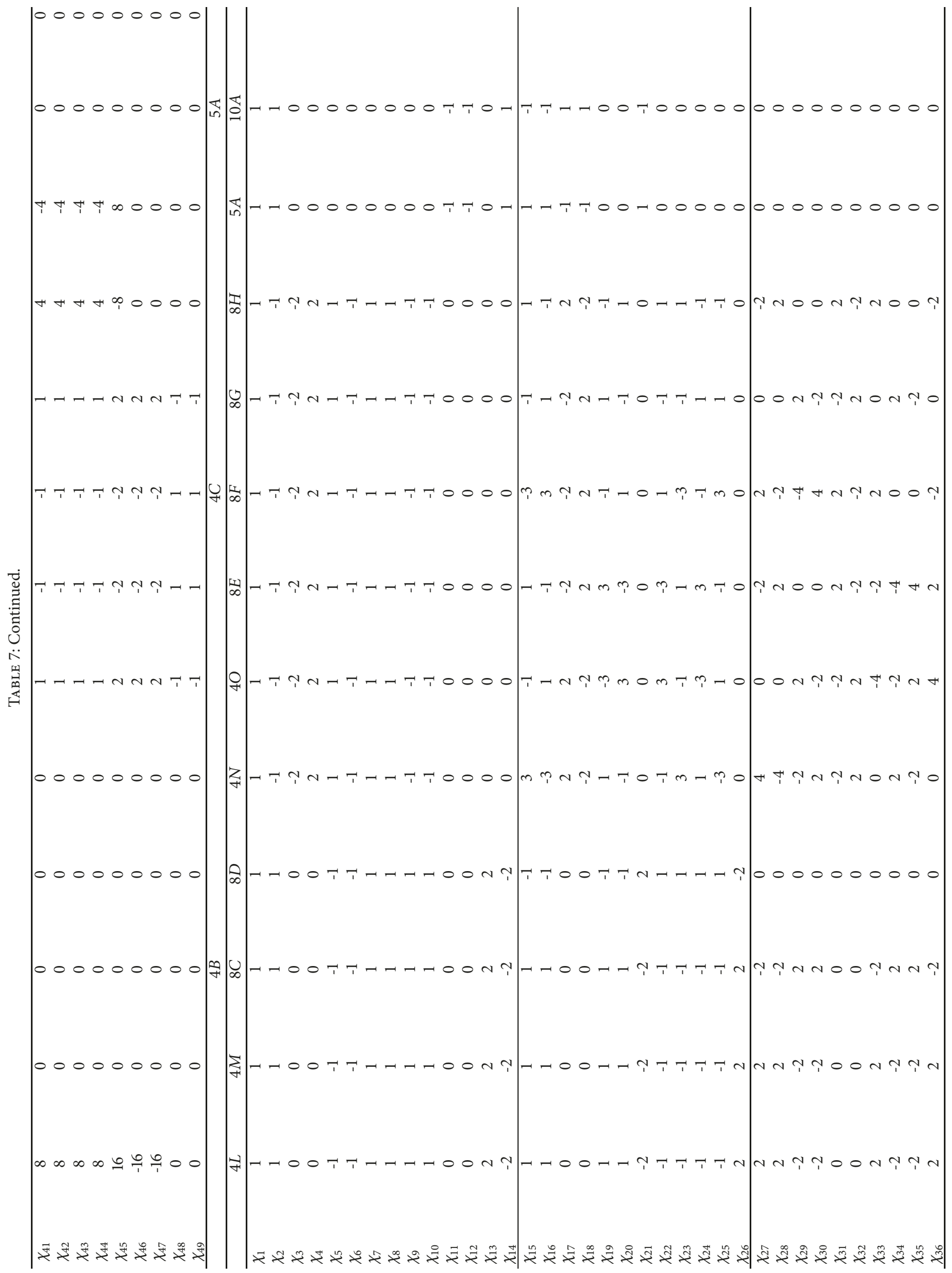




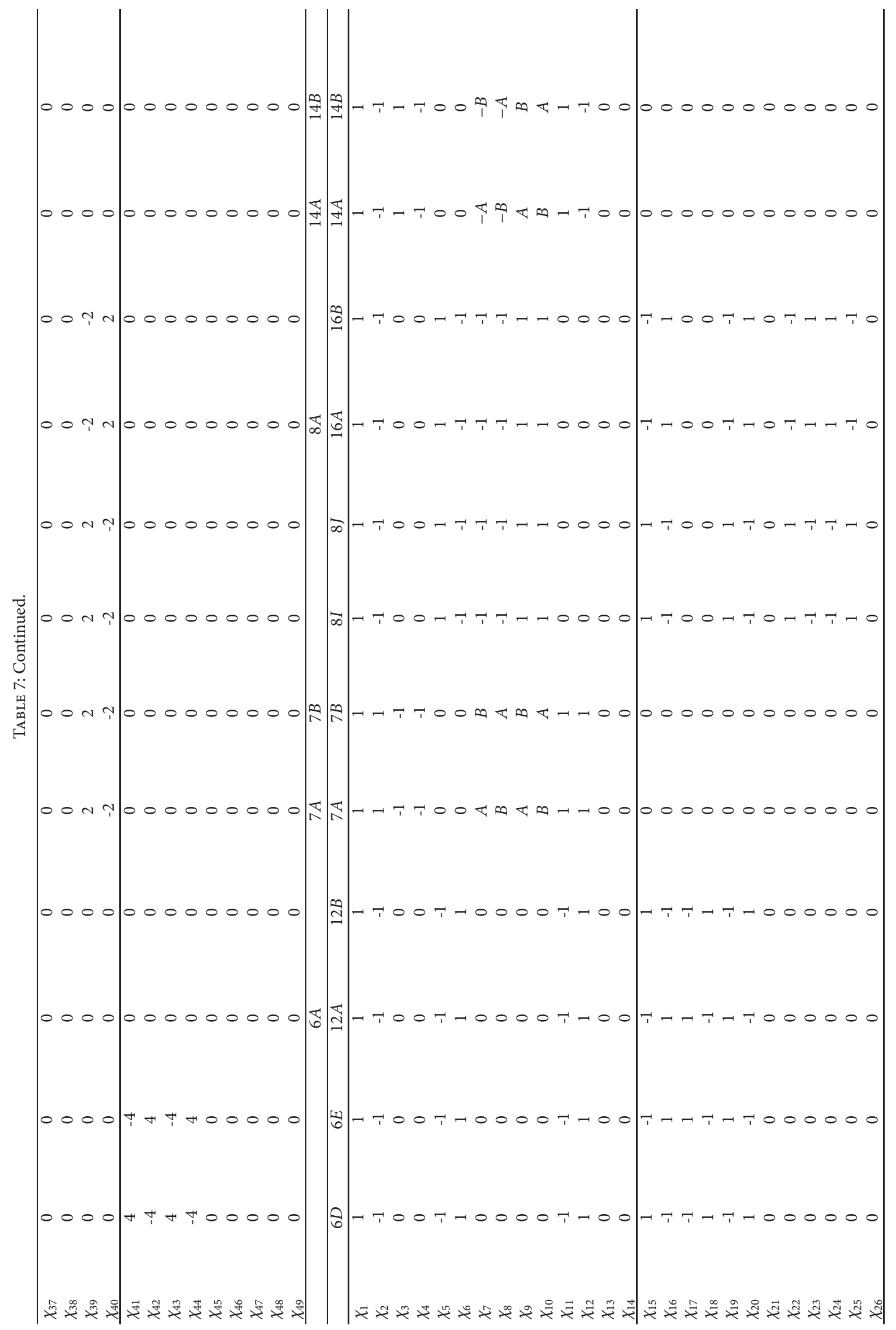




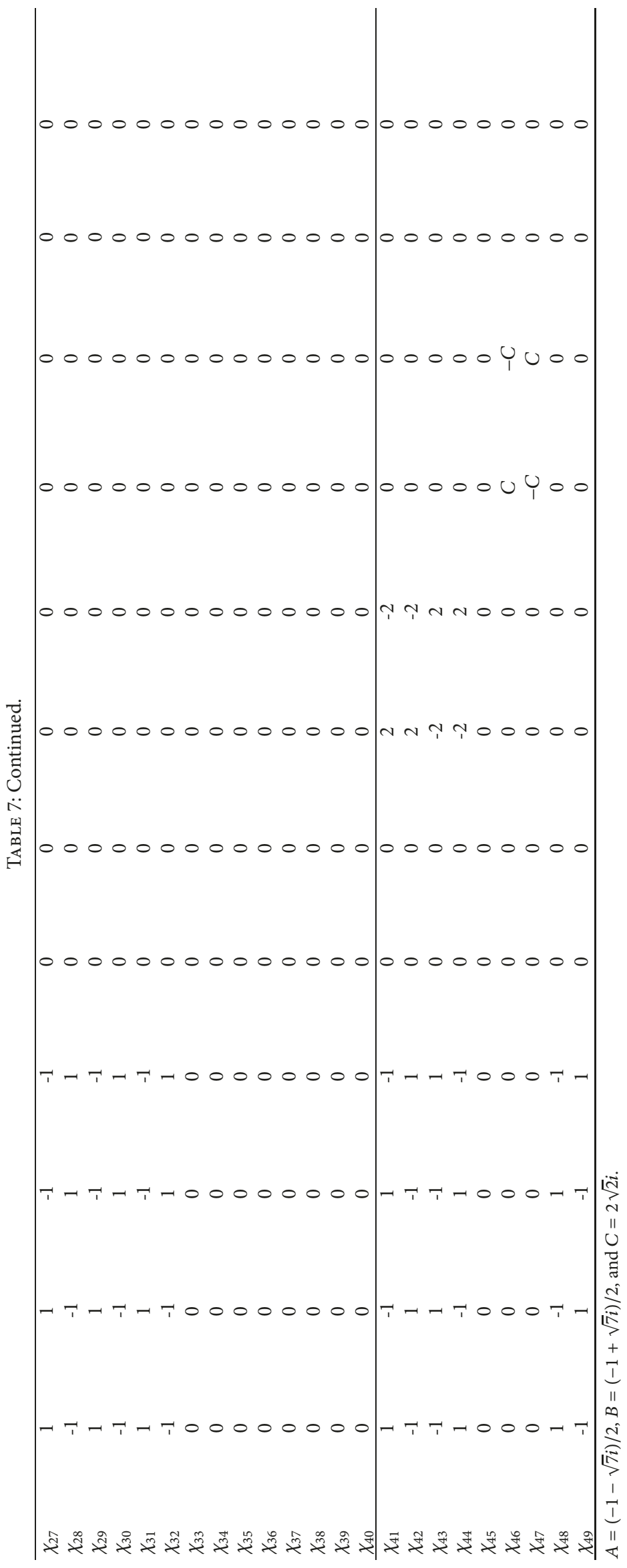


TABLE 8: The power maps of the elements of $2^{9}:\left(L_{3}(4): 2\right)$.

\begin{tabular}{|c|c|c|c|c|c|c|c|c|c|c|c|}
\hline$[g]_{G}$ & {$[x]_{\bar{G}}$} & 2 & 3 & 5 & 7 & {$[g]_{G}$} & {$[x]_{\bar{G}}$} & 2 & 3 & 5 & 7 \\
\hline \multirow{5}{*}{$1 A$} & $1 A$ & & & & & $2 A$ & $2 D$ & $1 A$ & & & \\
\hline & $2 A$ & $1 A$ & & & & & $2 E$ & $1 A$ & & & \\
\hline & $2 B$ & $1 A$ & & & & & $4 A$ & $2 B$ & & & \\
\hline & $2 C$ & $1 A$ & & & & & $4 B$ & $2 B$ & & & \\
\hline & & & & & & & $4 C$ & $2 B$ & & & \\
\hline \multirow[t]{8}{*}{$2 B$} & $2 F$ & $1 A$ & & & & $3 A$ & $3 A$ & & $1 A$ & & \\
\hline & $2 G$ & $1 A$ & & & & & $6 A$ & $3 A$ & $2 C$ & & \\
\hline & $4 D$ & $2 A$ & & & & & $6 B$ & $3 A$ & $2 A$ & & \\
\hline & $4 E$ & $2 A$ & & & & & $6 C$ & $3 A$ & $2 B$ & & \\
\hline & $4 F$ & $2 B$ & & & & & & & & & \\
\hline & $4 G$ & $2 B$ & & & & & & & & & \\
\hline & $4 H$ & $2 B$ & & & & & & & & & \\
\hline & $4 I$ & $2 B$ & & & & & & & & & \\
\hline \multirow[t]{4}{*}{$4 A$} & $4 J$ & $2 F$ & & & & $4 B$ & $4 L$ & $2 F$ & & & \\
\hline & $4 K$ & $2 F$ & & & & & $4 M$ & $2 F$ & & & \\
\hline & $8 A$ & $4 E$ & & & & & $8 C$ & $4 E$ & & & \\
\hline & $8 B$ & $4 F$ & & & & & $8 D$ & $4 I$ & & & \\
\hline \multirow[t]{6}{*}{$4 C$} & $4 N$ & $2 F$ & & & & $5 A$ & $5 A$ & & & $1 A$ & \\
\hline & $4 O$ & $2 F$ & & & & & $10 A$ & $5 A$ & & $2 A$ & \\
\hline & $8 E$ & $4 F$ & & & & & & & & & \\
\hline & $8 F$ & $4 F$ & & & & & & & & & \\
\hline & $8 G$ & $4 D$ & & & & & & & & & \\
\hline & $8 H$ & $4 F$ & & & & & & & & & \\
\hline \multirow[t]{4}{*}{$6 A$} & $6 D$ & $3 A$ & $2 D$ & & & $7 A$ & $7 A$ & & & $1 A$ & \\
\hline & $6 E$ & $3 A$ & $2 E$ & & & & & & & & \\
\hline & $12 \mathrm{~A}$ & $6 C$ & $4 C$ & & & & & & & & \\
\hline & $12 B$ & $6 C$ & $4 A$ & & & & & & & & \\
\hline \multirow[t]{4}{*}{$7 B$} & $7 B$ & & & & $1 A$ & $8 A$ & $8 I$ & $4 \mathrm{~J}$ & & & \\
\hline & & & & & & & $8 \mathrm{~J}$ & $4 J$ & & & \\
\hline & & & & & & & $16 A$ & $8 A$ & & & \\
\hline & & & & & & & $16 B$ & $8 A$ & & & \\
\hline $14 A$ & $14 A$ & $7 A$ & & & $2 D$ & $14 B$ & $14 B$ & $7 B$ & & & $2 D$ \\
\hline
\end{tabular}

$$
\begin{aligned}
& \theta_{3}=\sum_{j=23}^{232} \varphi_{j}, \quad \operatorname{deg}\left(\theta_{3}\right)=210 \\
& \theta_{4}=\sum_{j=233}^{512} \varphi_{j}, \quad \operatorname{deg}\left(\theta_{4}\right)=280 .
\end{aligned}
$$

Hence

$$
\phi_{2^{9}}=a_{1} \varphi_{1}+a_{2} \sum_{j=2}^{22} \varphi_{j}+a_{3} \sum_{j=23}^{232} \varphi_{j}+a_{4} \sum_{j=233}^{512} \varphi_{j}
$$

and therefore

$$
\left\langle\phi_{2^{9}}, \phi_{2^{9}}\right\rangle=a_{1}^{2}+21 a_{2}^{2}+210 a_{3}^{2}+280 a_{4}^{2}
$$

$$
\begin{aligned}
& =\frac{1}{512}\{\phi(1 A) \phi(1 A)+21 \phi(2 A) \phi(2 A) \\
& +210 \phi(2 B) \phi(2 B)+280 \phi(2 C) \phi(2 C)\},
\end{aligned}
$$

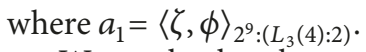

We apply the above results to some of the irreducible characters of $U_{6}(2): 2$ of small degrees, which in this case are $\phi_{1}=22 a, \phi_{2}=22 b, \phi_{3}=231 a, \phi_{4}=231 b, \phi_{5}=440 a$, and $\phi_{6}=440 b$. Their respective degrees are 22, 22, 231, 231, 440, and 440 . For $\phi_{1}$ we calculate that

$$
\begin{aligned}
& \left\langle\zeta, \phi_{1}\right\rangle_{2^{9}:\left(L_{3}(4): 2\right)} \\
& \quad=\frac{1}{512}\{22+21(-10)+210(6)+280(-2)\}=1 .
\end{aligned}
$$

Now $a_{1}+21 a_{2}+210 a_{3}+280 a_{4}=22$, since $\operatorname{deg} \phi_{1}=22$. Since $a_{1}=1$, we must have that $a_{2}=1$ and $a_{3}=a_{4}=0$. Note that $2^{9}:\left(L_{3}(4): 2\right)$ does not have irreducible characters of degree 22. We obtain that $\left(\phi_{1}\right)_{2^{9}:\left(L_{3}(4): 2\right.}=\chi_{1}+\chi_{15}$ if the partial fusion of $2^{9}:\left(L_{3}(4): 2\right)$ into $U_{6}(2): 2$ is taken into consideration. Similarly, for $\phi_{3}$ and $\phi_{5}$ we calculate that

$$
\begin{aligned}
& \left\langle\zeta, \phi_{3}\right\rangle_{2^{9}:\left(L_{3}(4): 2\right)} \\
& =\frac{1}{512}\{231+21(39)+210(7)+280(-9)\}=0
\end{aligned}
$$

and

$$
\begin{aligned}
& \left\langle\zeta, \phi_{5}\right\rangle_{2^{9}:\left(L_{3}(4): 2\right)} \\
& \quad=\frac{1}{512}\{440+21(120)+210(24)+280(8)\}=20 .
\end{aligned}
$$

Since the respective degrees of $\phi_{3}$ and $\phi_{5}$ are 231 and 440, we have to solve the equations (i) $a_{1}+21 a_{2}+210 a_{3}+280 a_{4}$ $=231$ and (ii) $a_{1}+21 a_{2}+210 a_{3}+280 a_{4}=440$, separately. If we are taking into account the fact that the set $\operatorname{Irr}(\bar{G})$ (see Table 7) does not have any irreducible characters of degrees 231 and 440 and also that $\left\langle\zeta, \phi_{3}\right\rangle_{2^{9}:\left(L_{3}(4): 2\right)}=0$ and $\left\langle\zeta, \phi_{5}\right\rangle_{2^{9}:\left(L_{3}(4): 2\right)}=20$, we deduce that the two sets of values $\left\{a_{1}=a_{4}=0, a_{2}=a_{3}=1\right\}$ and $\left\{a_{1}=20, a_{2}=10, a_{3}=\right.$ $\left.1, a_{4}=0\right\}$ are the only possibilities that satisfy equation (i) and (ii), respectively, hence we obtained that $\left(\phi_{3}\right)_{2^{9}:\left(L_{3}(4): 2\right)}=$ $\chi_{16}+\chi_{27}$ and $\left(\phi_{5}\right)_{2^{9}:\left(L_{3}(4): 2\right)}=\chi_{4}+\chi_{32}$. Similar computations were carried out to restrict the characters $\phi_{2}, \phi_{4}$, and $\phi_{6}$ to $\bar{G}$ and we found that $\left(\phi_{2}\right)_{2^{9}:\left(L_{3}(4): 2\right)}=\chi_{2}+\chi_{16},\left(\phi_{4}\right)_{2^{9}:\left(L_{3}(4): 2\right)}=$ $\chi_{15}+\chi_{28}$, and $\left(\phi_{6}\right)_{2^{9}:\left(L_{3}(4): 2\right)}=\chi_{3}+\chi_{31}$.

By making use of the values of $\phi_{1}, \phi_{2}, \phi_{3}, \phi_{4}, \phi_{5}$, and $\phi_{6}$ on the classes of $U_{6}(2): 2$ and the values of $\left(\phi_{1}\right)_{2^{9}:\left(L_{3}(4): 2\right)}$, $\left(\phi_{2}\right)_{2^{9}:\left(L_{3}(4): 2\right)},\left(\phi_{3}\right)_{2^{9}:\left(L_{3}(4): 2\right)},\left(\phi_{4}\right)_{2^{9}:\left(L_{3}(4): 2\right)},\left(\phi_{5}\right)_{2^{9}:\left(L_{3}(4): 2\right)}$, and $\left(\phi_{6}\right)_{2^{9}:\left(L_{3}(4): 2\right)}$ on the classes of $2^{9}:\left(L_{3}(4): 2\right)$ together with the partial fusion, the complete fusion map of $2^{9}:\left(L_{3}(4): 2\right)$ into $U_{6}(2): 2$ is given in Table 9 .

\section{Data Availability}

The data used to support the findings of this study are included within the article. 
TABle 9: The fusion of $2^{9}:\left(L_{3}(4): 2\right)$ into $U_{6}(2): 2$.

\begin{tabular}{|c|c|c|c|c|c|}
\hline$[g]_{L_{3}(4): 2}$ & {$[x]_{2^{9}:\left(L_{3}(4): 2\right)} \longrightarrow$} & {$[y]_{U_{6}(2): 2}$} & {$[g]_{L_{3}(4): 2}$} & {$[x]_{2^{9}:\left(L_{3}(4): 2\right)} \longrightarrow$} & {$[y]_{U_{6}(2): 2}$} \\
\hline \multirow[t]{5}{*}{$1 A$} & $1 A$ & $1 A$ & $2 A$ & $2 D$ & $2 D$ \\
\hline & $2 A$ & $2 A$ & & $2 E$ & $2 E$ \\
\hline & $2 B$ & $2 B$ & & $4 A$ & $4 G$ \\
\hline & $2 C$ & $2 C$ & & $4 B$ & $4 H$ \\
\hline & & & & $4 C$ & $4 I$ \\
\hline \multirow[t]{8}{*}{$2 B$} & $2 F$ & $2 B$ & $3 A$ & $3 A$ & $3 C$ \\
\hline & $2 G$ & $2 C$ & & $6 A$ & $6 G$ \\
\hline & $4 D$ & $4 A$ & & $6 B$ & $6 E$ \\
\hline & $4 E$ & $4 B$ & & $6 C$ & $6 F$ \\
\hline & $4 F$ & $4 C$ & & & \\
\hline & $4 G$ & $4 E$ & & & \\
\hline & $4 H$ & $4 F$ & & & \\
\hline & $4 I$ & $4 D$ & & & \\
\hline \multirow[t]{4}{*}{$4 A$} & $4 J$ & $4 C$ & $4 B$ & $4 L$ & $4 D$ \\
\hline & $4 K$ & $4 F$ & & $4 M$ & $4 F$ \\
\hline & $8 A$ & $8 A$ & & $8 C$ & $8 A$ \\
\hline & $8 B$ & $8 B$ & & $8 D$ & $8 C$ \\
\hline \multirow[t]{6}{*}{$4 B$} & $4 N$ & $4 H$ & $5 A$ & $5 A$ & $5 A$ \\
\hline & $4 O$ & $4 I$ & & $10 A$ & $10 A$ \\
\hline & $8 E$ & $8 F$ & & & \\
\hline & $8 F$ & $8 G$ & & & \\
\hline & $8 G$ & $8 D$ & & & \\
\hline & $8 H$ & $8 E$ & & & \\
\hline \multirow[t]{4}{*}{$6 A$} & $6 D$ & $6 K$ & $7 A$ & $7 A$ & $7 A$ \\
\hline & $6 E$ & $6 L$ & & & \\
\hline & $12 A$ & $12 K$ & & & \\
\hline & $12 B$ & $12 J$ & & & \\
\hline \multirow[t]{4}{*}{$7 B$} & $7 B$ & $7 A$ & $8 A$ & $8 I$ & $8 E$ \\
\hline & & & & $8 J$ & $8 F$ \\
\hline & & & & $16 A$ & $16 A$ \\
\hline & & & & $16 B$ & $16 B$ \\
\hline $14 \mathrm{~A}$ & $14 A$ & $14 A$ & $14 B$ & $14 B$ & $14 A$ \\
\hline
\end{tabular}

\section{Conflicts of Interest}

The authors declare that there are no conflicts of interest regarding the publication of this paper.

\section{Acknowledgments}

The second author acknowledges the financial support provided by the South African Department of Defense and the academic support from the 1st author towards the completion of his M.S. degree at the University of the Western Cape.

\section{References}

[1] J. H. Conway, R. T. Curtis, S. P. Norton, R. A. Parker, and R. A. Wilson, Atlas of Finite Groups, Oxford University Press, Oxford, UK, 1985.
[2] I. A. I. Suleiman and R. A. Wilson, "Covering and automorphism groups of $U_{6}(2)$," Quarterly Journal of Mathematics, vol. 48 , no. 192 , pp. 511-517, 1997.

[3] C. Parker and G. Stroth, "An identification theorem for $\operatorname{PSU}_{6}(2)$ and its automorphism group," 2011, https://arxiv.org/abs/ 1102.5392 .

[4] The GAP Group, "GAP -groups, algorithms, and programming," 2013, http://www.gap-system.org.

[5] B. Fischer, "Clifford-matrices," in Progress in Mathematics, G. O. Michler and C. Ringel, Eds., pp. 1-16, Birkhauser, Basel, Switzerland, 1991.

[6] A. B. M. Basheer and J. Moori, "a survey on Clifford-Fischer theory," in London Mathematical Society Lecture Notes Series, vol. 422, pp. 160-172, Cambridge University Press, 2015.

[7] R. L. Fray, R. L. Monaledi, and A. L. Prins, "Fischer-Clifford matrices of $2^{8}:\left(U_{4}(2): 2\right)$ as a subgroup of $O_{10}^{+}(2)$," Afrika Matematika, vol. 27, no. 7-8, pp. 1295-1310, 2016. 
[8] J. Moori, "On certain groups associated with the smallest Fischer group," Journal of the London Mathematical Society, vol. 23, no. 1, pp. 61-67, 1981.

[9] J. Moori and Z. E. Mpono, "The Fischer-Clifford matrices of the group $2^{6}: \mathrm{SP}_{6}(2)$," Quaestiones Mathematicae, vol. 22, pp. 257$298,1999$.

[10] A. L. Prins, "The Fischer-Clifford matrices and character table of the maximal subgroup $2^{9}:\left(L_{3}(4): S_{3}\right)$ of $U_{6}(2): S_{3}$," Bulletin of the Iranian Mathematical Society, vol. 42, no. 5, pp. 1179-1195, 2016.

[11] W. Bosma and J. J. Cannon, Handbook of Magma Functions, Department of Mathematics, University of Sydney, 1994.

[12] R. A. Wilson, P. Walsh, J. Tripp et al., ATLAS of Finite Group Representations, 1999, http://brauer.maths.qmul.ac.uk/Atlas/v3/.

[13] T. Connor and D. Leemans, "An atlas of subgroup lattices of finite almost simple groups," Ars Mathematica Contemporanea, vol. 8, no. 2, pp. 259-266, 2015.

[14] Z. Mpono, Fischer-Clifford Theory and Character Tables of Group Extensions [PhD Thesis], University of Natal, 1998.

[15] J. J. Cannon, "An introduction to the group theory language CAYLEY," in Computational Group Theory, M. Atkinson, Ed., pp. 145-183, Academic Press, San Diego, Calif, USA, 1984.

[16] F. Ali, Fischer-Clifford Theory for Split and Non-Split Group Extensions [Phd Thesis], University of Natal, 2001.

[17] D. Gorenstein, Finite Groups, Harper and Row Publishers, New York, NY, USA, 1968.

[18] T. T. Seretlo, Fischer Clifford Matrices and Character Tables of Certain Groups Associated with Simple Groups $\mathrm{O}_{10}^{+}$, HS and Ly [PhD Thesis], University of KwaZulu Natal, 2011.

[19] R. L. Fray and A. L. Prins, "On the inertia groups of the maximal subgroup $2^{7}: \operatorname{SP}(6,2)$ in $\operatorname{Aut}\left(\mathrm{Fi}_{22}\right)$," Quaestiones Mathematicae, vol. 38, no. 1, pp. 83-102, 2015. 


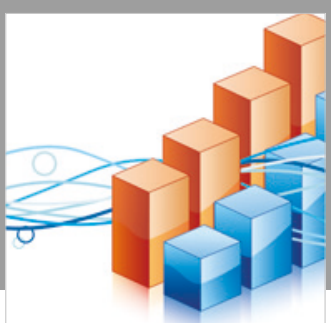

Advances in

Operations Research

\section{-n-m}
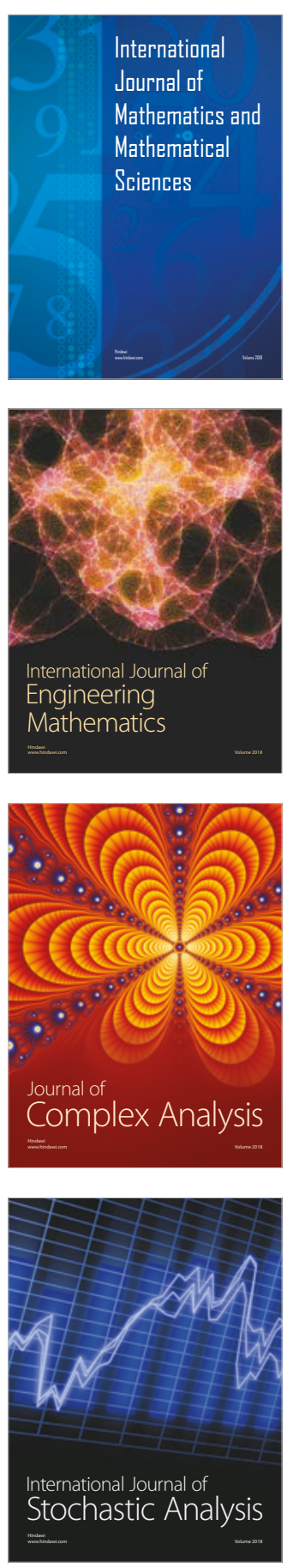
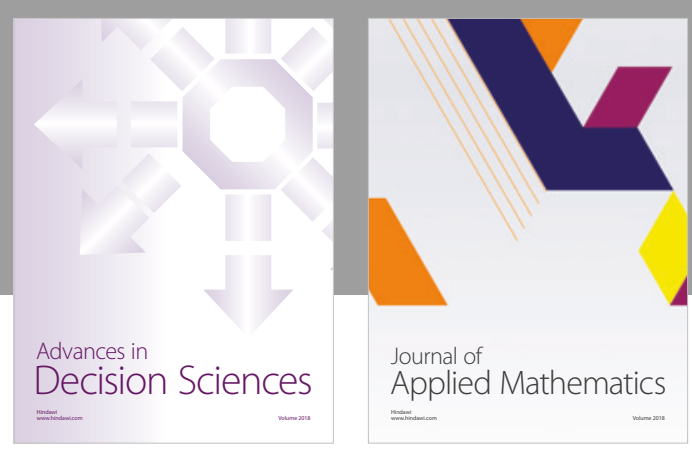

Journal of

Applied Mathematics
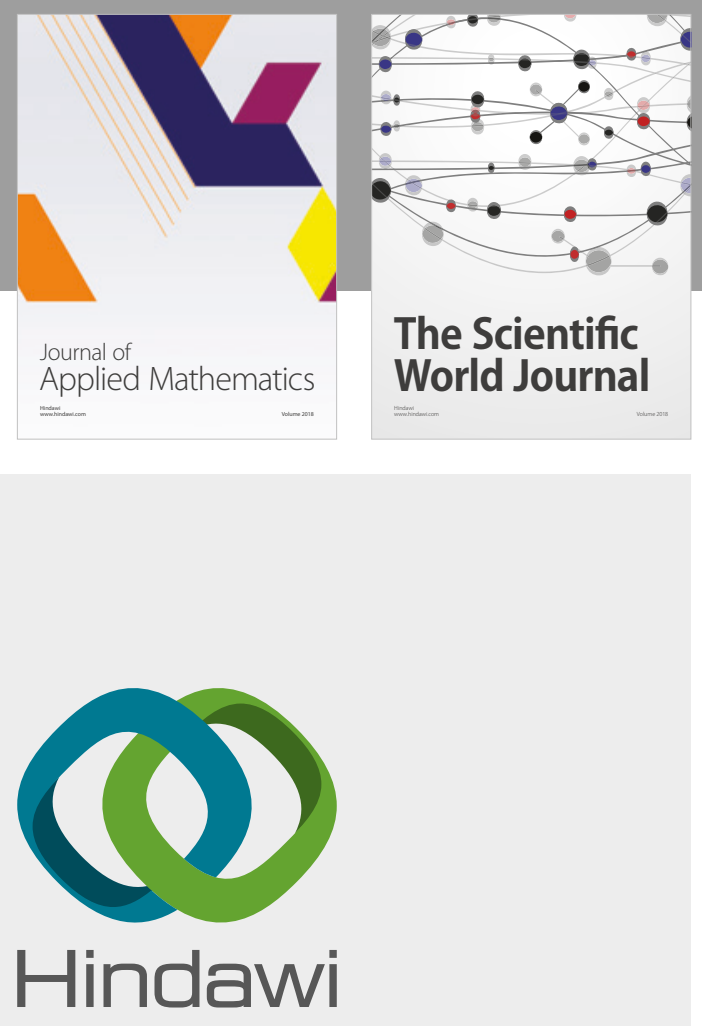

Submit your manuscripts at

www.hindawi.com

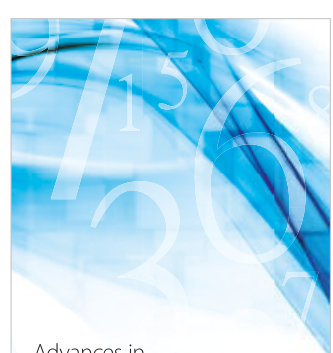

Advances in
Numerical Analysis
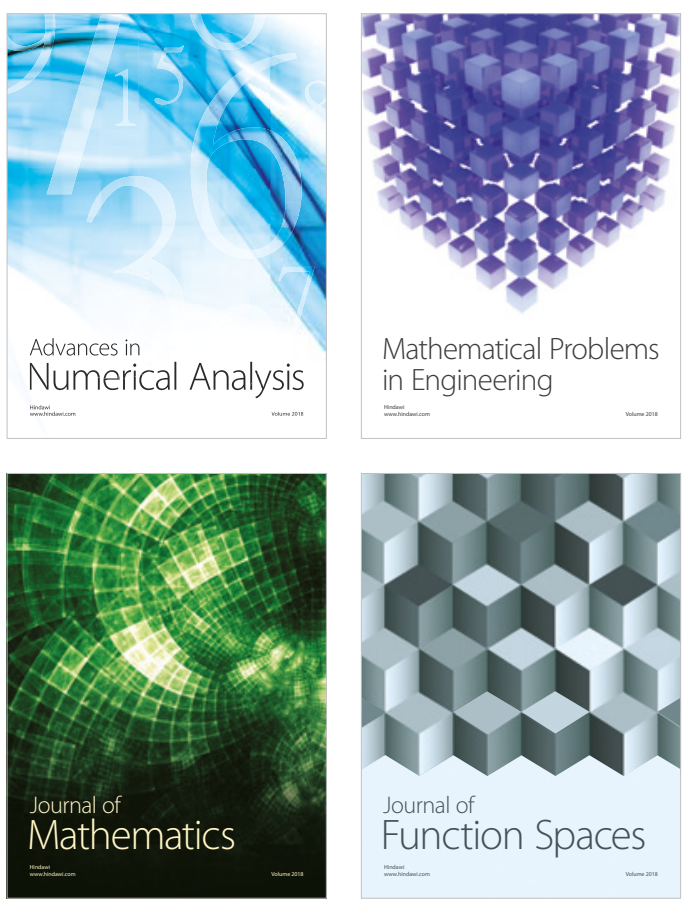

Mathematical Problems in Engineering

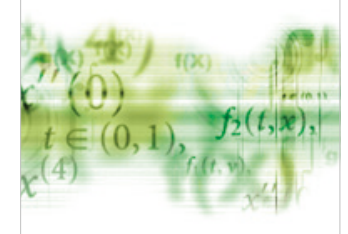

International Journal of

Differential Equations

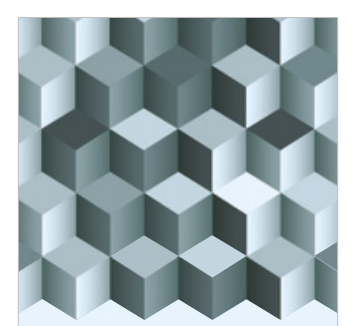

Journal of

Function Spaces

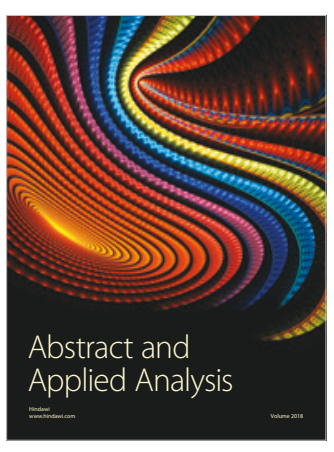

The Scientific

World Journal

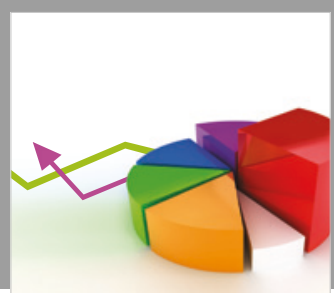

Journal of

Probability and Statistics
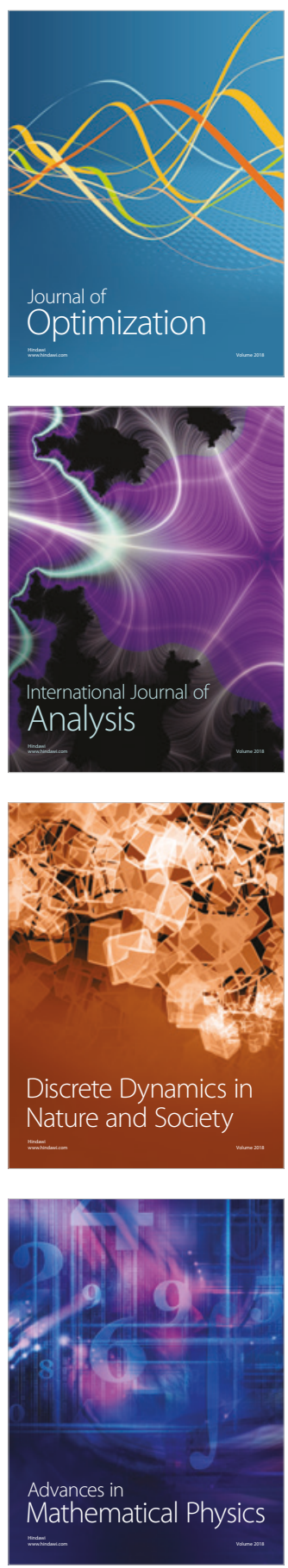